\title{
Multiscale computational first order homogenization of thick shells for the analysis of out-of-plane loaded masonry walls
}

\author{
Massimo Petracca ${ }^{\mathrm{a}, \mathrm{b}, \mathrm{c}, *}$, Luca Pelà $\grave{a}^{\mathrm{a}, \mathrm{b}}$, Riccardo Rossi ${ }^{\mathrm{a}, \mathrm{b}}$, Sergio Oller ${ }^{\mathrm{a}, \mathrm{b}}$, Guido \\ Camata $^{c}$, Enrico Spacone ${ }^{\mathrm{c}}$ \\ ${ }^{a}$ Department of Civil and Environmental Engineering, Technical University of Catalonia (UPC), \\ Barcelona 08034, Spain \\ ${ }^{b}$ CIMNE - Centre Internacional de Metodes Numerics en Enginyeria, Technical University of \\ Catalonia (UPC), Barcelona 08034, Spain \\ ${ }^{c}$ Department of Engineering, University "G.d'Annunzio" of Chieti and Pescara, Pescara 65127, Italy
}

\begin{abstract}
This work presents a multiscale method based on computational homogenization for the analysis of general heterogeneous thick shell structures, with special focus on periodic brick-masonry walls. The proposed method is designed for the analysis of shells whose micro-structure is heterogeneous in the in-plane directions, but initially homogeneous in the shell-thickness direction, a structural topology that can be found in single-leaf brick masonry walls. Under this assumption, this work proposes an efficient homogenization scheme where both the macro-scale and the micro-scale are described by the same shell theory. The proposed method is then applied to the analysis of out-of-plane loaded brick-masonry walls, and compared to experimental and micro-modeling results.
\end{abstract}

Keywords: Computational Multiscale Homogenization, Representative Volume Element (RVE), Shell, Masonry, Periodic Micro-structure, Strain Localization

\section{Introduction}

Masonry is an ancient building material that have been extensively used throughout the history, and it is still used nowadays. Being the main building technique adopted in historical constructions, a deep understanding of masonry mechanical behavior is therefore of primary importance for the preservation of our cultural heritage. However, the formulation of phenomenological constitutive equations for heterogeneous materials such as masonry is still a challenge $[1,2,3]$. The evolution of the structural response strongly depends on complex micro-structural phenomena such as damaged-induced anisotropy and localization of deformation in the micro-structure. It is therefore difficult to account for the influence that this evolving micro-structure has on the overall macroscopic

\footnotetext{
* corresponding author

Email addresses: mpetracca@cimne.upc.edu (Massimo Petracca), luca.pela@upc.edu (Luca Pelà), rrossi@cimne.upc.edu (Riccardo Rossi), oller@cimne.upc.edu (Sergio Oller), g.camata@unich.it (Guido Camata), espacone@unich.it (Enrico Spacone) 
behavior, especially when strain localization occurs in the micro-structure, leading to complex dissipative mechanisms at the structural scale. Advanced numerical methods are therefore attractive tools to understand and predict the behavior of masonry up to its complete failure, allowing to estimate the residual strength and safety of structures. A popular method commonly used nowadays to study masonry, accounting for its heterogeneous micro-structure, is micro-/meso-modeling, also known as Direct Numerical Simulation (DNS), $[4,5,6,7,8,9,10,11]$. In this approach, the complete micro-structure (or a simplified version of it) is directly modeled, thus the complex interaction between the masonry constituents is naturally taken into account. However, this approach leads to complex models and high computational costs. Therefore DNS is suitable to model in detail small structural members, but their usage soon becomes unaffordable for large scale structures. On the contrary, macro-modeling regards masonry as an equivalent homogeneous continuum, without making any distinction between units and joints in the discrete model $[1,2,12]$. This method possesses some intrinsic difficulties related to identification of mechanical properties and definition of reliable and predictive phenomenological failure criteria. Nevertheless, macro-modeling is still the preferred option for the analysis of large-scale structures, thanks to its affordable computational cost. An example of masonry macro-modeling for shells has been proposed in [13].

In-between macro- and micro-modeling techniques, Computational Homogenization Meth-

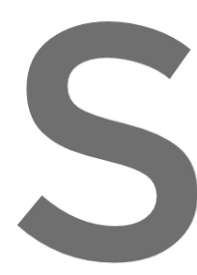
ods (CHM) have recently emerged as a promising tool joining their advantages [14. 15. 16

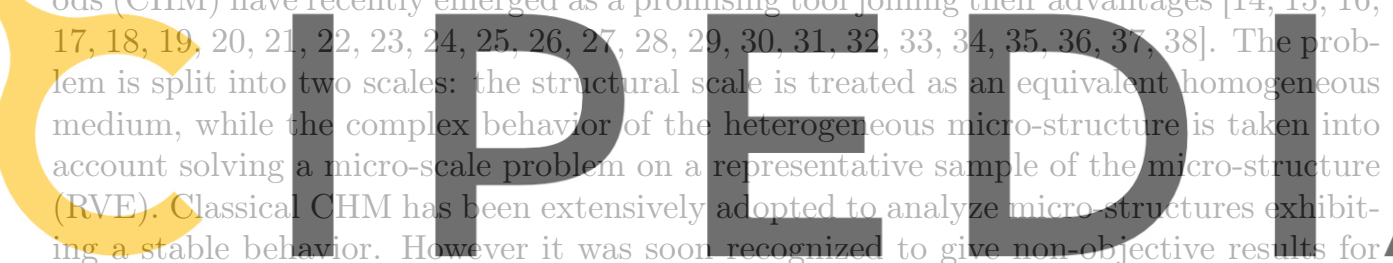

strain-softening materials, with the homogenized response not converging to a unique

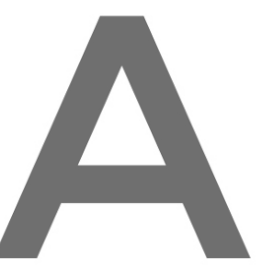

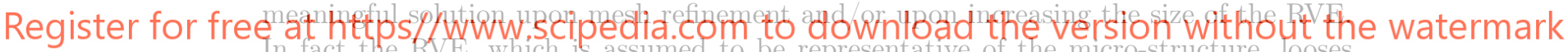

its representativeness upon localization of deformation. It should be noted that this.

issue is not directiy related to the CHIM itself, but stems from the use of a first order continuum. In the last years, some enhancements of the classical first order CHM were proposed, and a review on most recent developments can be found in [39] and in the references therein. Some methods regularize the response of the RVE adopting higher order continua at the macro-scale, so that a material characteristic length is naturally accounted for $[19,21,22,16,17,36,14]$. Others, known as continuous-discontinuous methods, up-scale the micro-structural response to a discontinuity at the structural scale $[23,24,25,26,27,29,40,15]$. In [41] an extension of the fracture-energy-based regularization to the homogenization problem has been proposed, and applied to the simulation of in-plane loaded masonry shear walls, allowing the use of a standard continuum theory, while ensuring a proper dissipation at the structural level.

Computational Homogenization for shell-like structures is an emerging branch of Computational Homogenization Methods. The need for this kind of homogenization stems from the fact that the size of the micro-structure has the same order of magnitude of the structure thickness, so that a standard 3D continuum homogenization cannot be applied, at least with respect to the thickness direction. In the last years some works have been done for thin structured shells in generic structural applications, with elastic or inelastic 
material behavior, with or without geometric non-linearity [42, 43, 44, 45, 46]. In these approaches, a 3D solid RVE discretizing the whole micro-structure in the thickness direction, is coupled to the macro-scale shell model. Applications of this concept to the specific case of masonry structures can be found in [25, 26]. In those works, material non-linearity was considered, and the homogenization was based on a Kirchhoff-Love (thin) shell theory, while the nonlinear transverse shear behavior was obtained by means of a separated model (i.e. not directly from the RVE calculations).

The present work proposes a new homogenization procedure for shell-like structures, based on Reissner-Mindlin (thick) shell theory. This approach is tailored to the homogenization of micro-structures that are heterogeneous in the in-plane directions, but (initially) homogeneous in the thickness direction (i.e. an extrusion of a heterogeneous surface in the thickness direction). With this assumption, both the macro-scale domain and the RVE domain can be accurately described according to shell theories. ReissnerMindlin shell theory is based on the hypothesis that the initially straight normals to the mid-plane of the shell remain straight after deformation, even though not necessarily perpendicular to the mid-plane. If the micro-structure is homogeneous in the thickness direction, it is reasonable to assume that the previously mentioned hypothesis holds. This assumption reduces the range of applicability of this method, with respect to those methods using a 3D RVE, but it greatly simplifies the macro-micro scale transition (both scale share the same theory), and it reduces the computational cost of the RVE

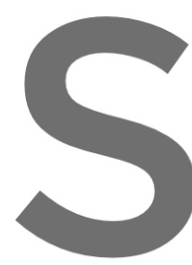
calculations. The Masonry Walls out-of-plane behav experimental stu to assess the cap erences therein
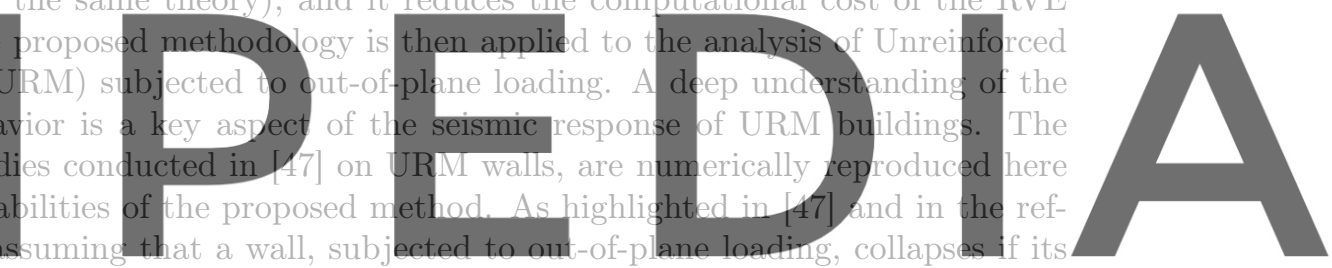

bending strength is exceeded is extremely conservative to assess its seismic response, as approaches the thickness of the wall. Therefore accurate constitutive models are required to numerically reproduce the out-of-plane behavior of masonry. The proposed methodology has proven to be rather accurate in reproducing the key aspects of the out-of-plane behavior of URM emerged from the experimental tests. 


\section{Computational homogenization framework}

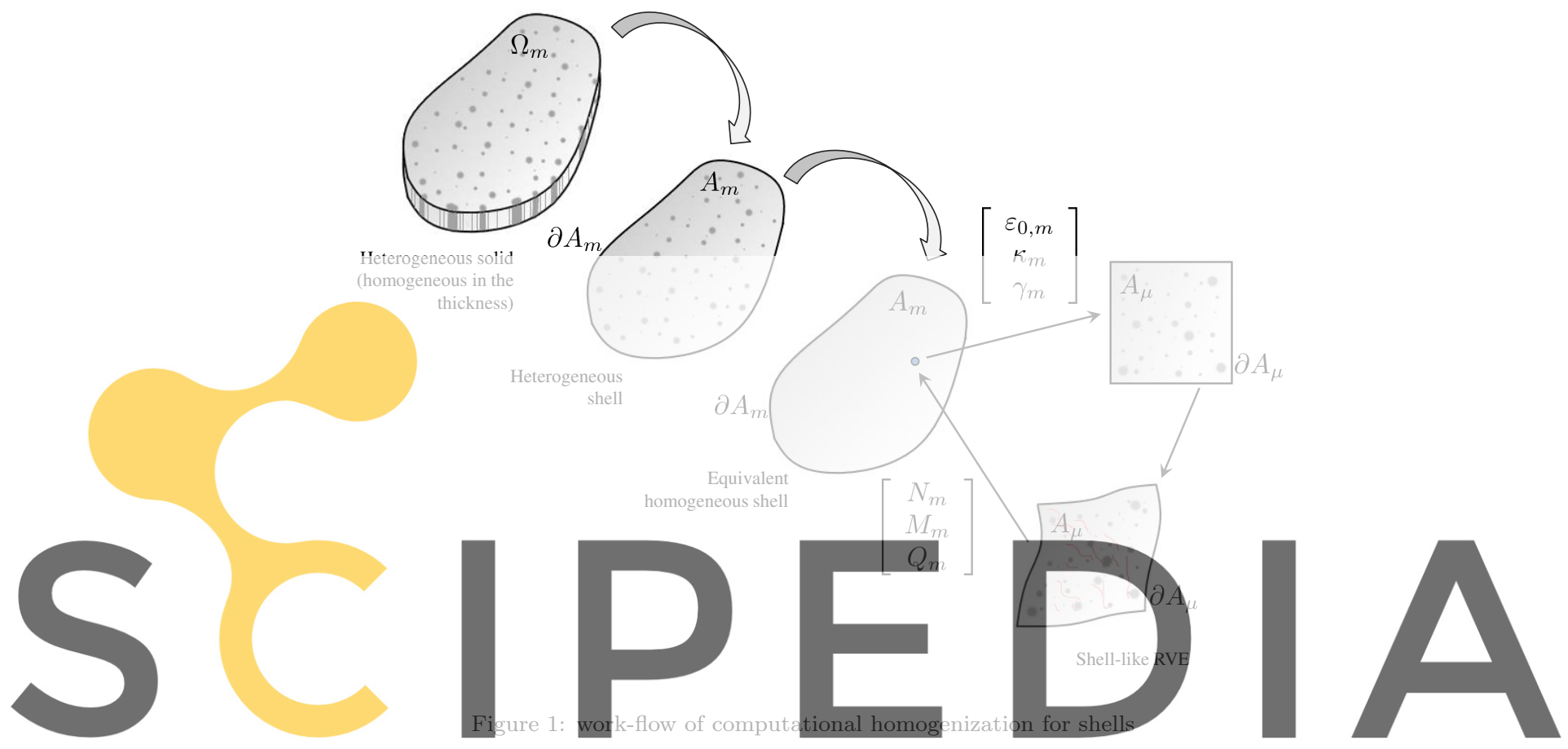

Register for free at https//www.scipedia.com to download the version without the watermark

This section gives the main concepts and basic equations of the proposed computational homogenization framework, where classical first order homogenization is extended to the case of shell theory. The following derivations assume small strain theory.

The main work-flow of the proposed CHM for shells can be represented as in Figure 1.

The proposed method is formally identical to the classical CHM for 2D and 3D continua [41]. The main difference is that now the continuum strain and stress tensors should be replaced with the generalized strains and generalized stresses typical of shell kinematics, as described in Section 2.1.

The homogenization process can be seen as a 3-step procedure:

1. Down-scaling or macro-micro transition. The macroscopic generalized strain, at any point of the macroscopic domain, are sent to the micro-scale, and used to define RVE boundary conditions;

2. Solution of the micro-scale Boundary Value Problem (BVP). The micro-structural problem is solved on the RVE domain. Any numerical method can be used. FEM is considered in this work;

3. Up-scaling or micro-macro transition. The macro-scale generalized stresses are obtained via homogenization procedures. 


\subsection{Shell kinematics}

From now on, any generalized quantity related to the shell theory is identified with a superimposed hat $\widehat{(\cdot)}$

The generalized unknown vector in the local coordinate system of the shell is defined as:

$$
\widehat{\boldsymbol{u}}=\left[\begin{array}{lll}
\boldsymbol{u}_{0} & u_{z} & \boldsymbol{\theta}
\end{array}\right]^{T}=\left[\begin{array}{lllll}
u_{x} & u_{y} & u_{z} & \theta_{x} & \theta_{y}
\end{array}\right]^{T}
$$

where $\boldsymbol{u}_{0}=\left[\begin{array}{ll}u_{x} & u_{y}\end{array}\right]^{T}$ are the in-plane displacements, $u_{z}$ is the out-of-plane displacement, and $\boldsymbol{\theta}=\left[\begin{array}{ll}\theta_{x} & \theta_{y}\end{array}\right]^{T}$ are the out-of-plane rotations about the local $x$ and $y$ axes respectively.

According to the first order shear deformation theory of Reissner-Mindlin, the generalized strains of the shell are defined as:

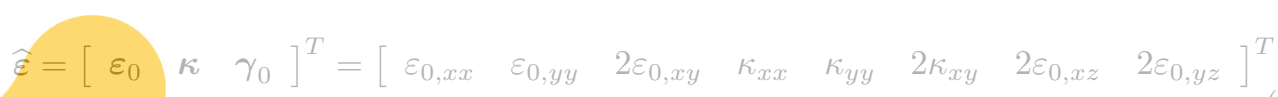

The in-plane strain tensor due to membrane actions is defined as:

$$
\varepsilon_{0}=\nabla^{s} u_{0}
$$

\section{The curvature tensor due to bending actions is defined as:}
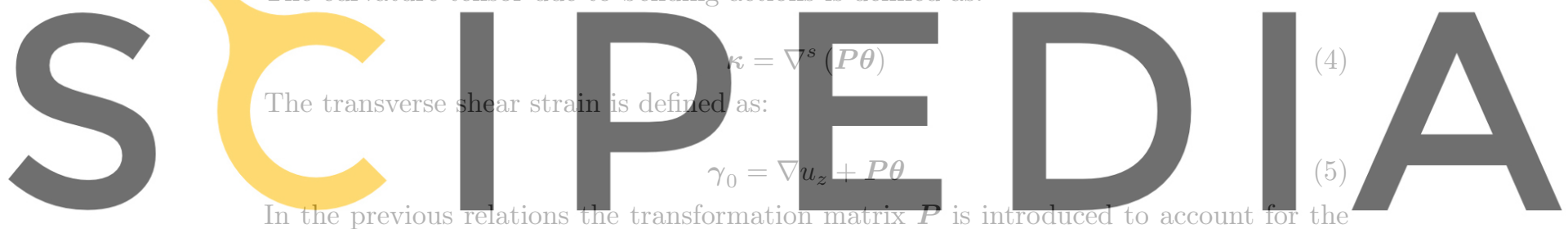

sign convention about rotations:

Register for free at https//www.scipedia.com to download the version without the watermark

$$
\begin{aligned}
& P=\left[\begin{array}{cc}
0 & 1 \\
-1 & 0
\end{array}\right] \\
& \boldsymbol{P} \boldsymbol{\theta}=\left[\begin{array}{c}
\theta_{y} \\
-\theta_{x}
\end{array}\right]
\end{aligned}
$$

Therefore the generalized strains of the shell can be rewritten as:

$$
\widehat{\boldsymbol{\varepsilon}}=\left[\begin{array}{c}
\varepsilon_{0, x x} \\
\varepsilon_{0, y y} \\
2 \varepsilon_{0, x y} \\
\kappa_{x x} \\
\kappa_{y y} \\
2 \kappa_{x y} \\
2 \varepsilon_{0, x z} \\
2 \varepsilon_{0, y z}
\end{array}\right]=\left[\begin{array}{c}
\frac{\partial u_{x}}{\partial x} \\
\frac{\partial u_{y}}{\partial y} \\
\frac{\partial u_{x}}{\partial y}+\frac{\partial u_{y}}{\partial x} \\
\frac{\partial \theta_{y}}{\partial x} \\
-\frac{\partial \theta_{x}}{\partial y} \\
\frac{\partial \theta_{y}}{\partial y}-\frac{\partial \theta_{x}}{\partial x} \\
\frac{\partial u_{z}}{\partial x}+\theta_{y} \\
\frac{\partial u_{z}}{\partial y}-\theta_{x}
\end{array}\right]
$$


2.2. Shell constitutive response: through-the-thickness integrated cross section

With the shell generalized strains $\widehat{\varepsilon}$ at hand, it is possible to calculate the constitutive response of the shell to obtain the generalized stresses:

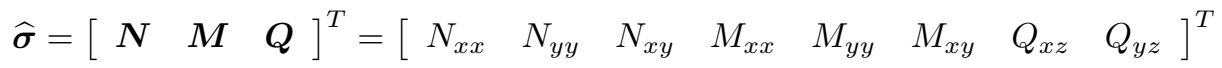

where $\boldsymbol{N}=\left[\begin{array}{lll}N_{x x} & N_{y y} & N_{x y}\end{array}\right]^{T}$ is the membrane force vector per unit length, $\boldsymbol{M}=$ $\left[\begin{array}{lll}M_{x x} & M_{y y} & M_{x y}\end{array}\right]^{T}$ is the bending/twisting moment vector per unit length, and $\boldsymbol{Q}=$ $\left[\begin{array}{ll}Q_{x z} & Q_{y z}\end{array}\right]^{T}$ is the transverse shear force vector per unit length. A very simple and general way to obtain the shell constitutive response is by means of a through-thethickness integration of standard continuum constitutive models.

Defining $\zeta$ as the through-the-thickness coordinate, ranging from $-H / 2$ to $H / 2$ ( $H$ being the thickness of the shell), the strain at each coordinate $\zeta$ can be defined as:

$$
\varepsilon_{(\zeta)}=\varepsilon_{0}+\zeta \kappa+\gamma_{0}
$$

or in matrix form:

$$
\varepsilon_{(\zeta)}=H_{\varepsilon(\zeta)} \widehat{\varepsilon}
$$
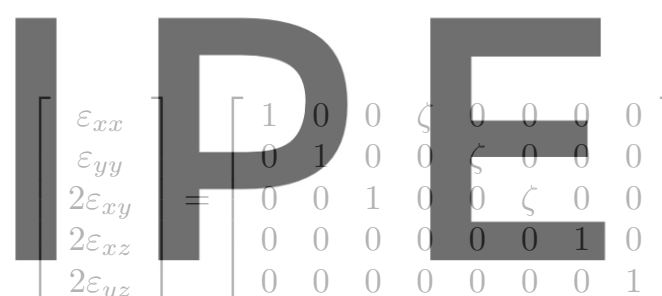

(11)

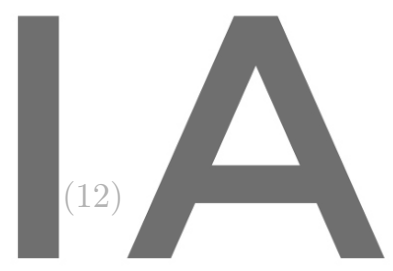

Register for free at https//www.scipedia.com to download the zersion without the watermark

Then the stress tensor and the constitutive tangent operator at any coordinate $\zeta$ in the

thickness direction can be calculated from any constitutive model:

$$
\boldsymbol{\sigma}_{(\zeta)}=f\left(\varepsilon_{(\zeta)}\right), \boldsymbol{C}_{(\zeta)}=\frac{\partial \boldsymbol{\sigma}_{(\zeta)}}{\partial \varepsilon_{(\zeta)}}
$$

The generalized stresses and the tangent constitutive matrix for the shell can be finally integrated through the thickness:

$$
\begin{gathered}
\widehat{\boldsymbol{\sigma}}=\int_{-\frac{H}{2}}^{\frac{H}{2}} \boldsymbol{H}_{\sigma(\zeta)} \boldsymbol{\sigma}_{(\zeta)} \mathrm{d} \zeta \\
{\left[\begin{array}{c}
N_{x x} \\
N_{y y} \\
N_{x y} \\
M_{x x} \\
M_{y y} \\
M_{x y} \\
Q_{x z} \\
Q_{y z}
\end{array}\right]=\int_{-\frac{H}{2}}^{\frac{H}{2}}\left[\begin{array}{ccccc}
1 & 0 & 0 & 0 & 0 \\
0 & 1 & 0 & 0 & 0 \\
0 & 0 & 1 & 0 & 0 \\
\zeta & 0 & 0 & 0 & 0 \\
0 & \zeta & 0 & 0 & 0 \\
0 & 0 & \zeta & 0 & 0 \\
0 & 0 & 0 & k & 0 \\
0 & 0 & 0 & 0 & k
\end{array}\right]\left[\begin{array}{c}
\sigma_{x x} \\
\sigma_{y y} \\
\sigma_{x y} \\
\sigma_{x z} \\
\sigma_{y z}
\end{array}\right] \mathrm{d} \zeta} \\
\\
\end{gathered}
$$




$$
\widehat{\boldsymbol{C}}=\frac{\partial \widehat{\boldsymbol{\sigma}}}{\partial \widehat{\varepsilon}}=\int_{-\frac{H}{2}}^{\frac{H}{2}} \boldsymbol{H}_{\sigma(\zeta)} \boldsymbol{C}_{(\zeta)} \boldsymbol{H}_{\varepsilon(\zeta)} \mathrm{d} \zeta
$$

where $k$ is the transverse shear correction factor [48].

It should be noted that in Eq. (13) the continuum stress tensor $\boldsymbol{\sigma}_{(\zeta)}$ at a certain $\zeta$ coordinate in the thickness direction, is obtained as a function of the continuum strain tensor $\varepsilon_{(\zeta)}$ at the same $\zeta$ coordinate. However, the strain tensor $\varepsilon_{(\zeta)}$, as defined in Eq. (12), is lacking the thickness strain component $\varepsilon_{z z}$, since the standard shell kinematics does not provide it. A simple way to cope with this issue would be to obtain $\boldsymbol{\sigma}_{(\zeta)}^{P S}$, the plane-stress part of $\boldsymbol{\sigma}_{(\zeta)}$, through any general (non-linear) plane stress constitutive law $f^{P S}(\cdot)$, while considering $\boldsymbol{\sigma}_{(\zeta)}^{T S}$, the transverse shear part of $\boldsymbol{\sigma}_{(\zeta)}$, elastic:

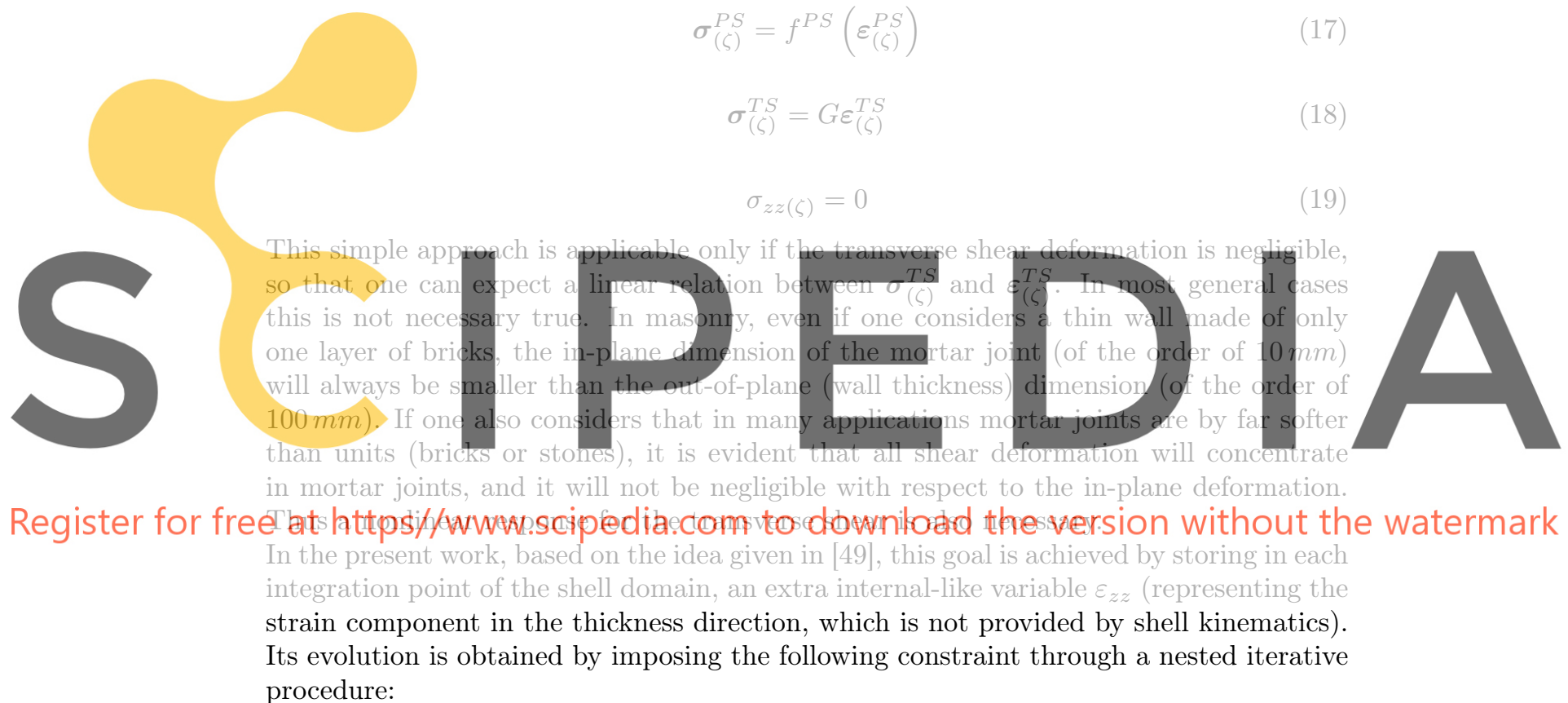

$$
\text { find } \varepsilon_{z z} \text { such that } \int_{-\frac{H}{2}}^{\frac{H}{2}} \sigma_{z z(\zeta)} \mathrm{d} \zeta=0
$$

In this way, the out-of-plane normal stress is not forced to be zero at every depth in the thickness, but only its integral (the stress resultant $N_{z z}$ ) is constraint to be zero, as required by the thick shell theory.

This procedure allows to use full 3D constitutive models along the thickness of the shell. The generalized stresses and the generalized constitutive tensor can then be obtained using Eq. (14) and Eq. (16), respectively. Indeed Eq. (14) and Eq. (16) are integrals along the thickness direction, therefore a suitable integration scheme should be provided. Note that this is an approximation of the real 3D behavior. The out-of-plane strain component, $\varepsilon_{z z}$, is assumed constant in the thickness direction, and is calculated locally at each gausspoint of the shell element. The iterative procedure in Eq. (20) can be avoided in case 
of other shell formulations whose kinematics can provide the thickness stretch, such as 3D-shells for example.

\subsection{Down-scaling or macro-micro transition and constraint conditions}

The macroscopic generalized strain $\widehat{\boldsymbol{\varepsilon}}_{m}$, in each point $\boldsymbol{x}_{m}$ of the macro-scale domain and at each instant $t$, can be obtained as the surface average of the microscopic generalized strain field $\widehat{\varepsilon}_{\mu}$ defined at each point $\boldsymbol{x}_{\mu}$ of the micro-scale domain and at each instant $t$ :

$$
\widehat{\boldsymbol{\varepsilon}}_{m}\left(\boldsymbol{x}_{m}, t\right)=\frac{1}{A_{\mu}} \int_{\Omega_{\mu}} \widehat{\boldsymbol{\varepsilon}}_{\mu}\left(\boldsymbol{x}_{\mu}, t\right) \mathrm{d} A
$$

where $A_{\mu}$ is the surface area of the shell domain at the micro-scale.

To account for the heterogeneity of the micro-structure, the microscopic displacement field $\widehat{\boldsymbol{u}}_{\mu}$ can be additively split into a coarse scale contribution $\widehat{\boldsymbol{u}}_{m}$ (arising from the macroscopic generalized strain field), and a fine scale contribution $\widehat{\tilde{u}}_{\mu}$ (obtained from the solution of the micro-scale problem). Accordingly, the microscopic generalized strain field is split in the same way:

$$
\widehat{\varepsilon}_{\mu}\left(x_{\mu}, t\right)=\widehat{\varepsilon}_{m}\left(x_{m}, t\right)+\widehat{\widetilde{\varepsilon}}_{\mu}\left(x_{\mu}, t\right)
$$
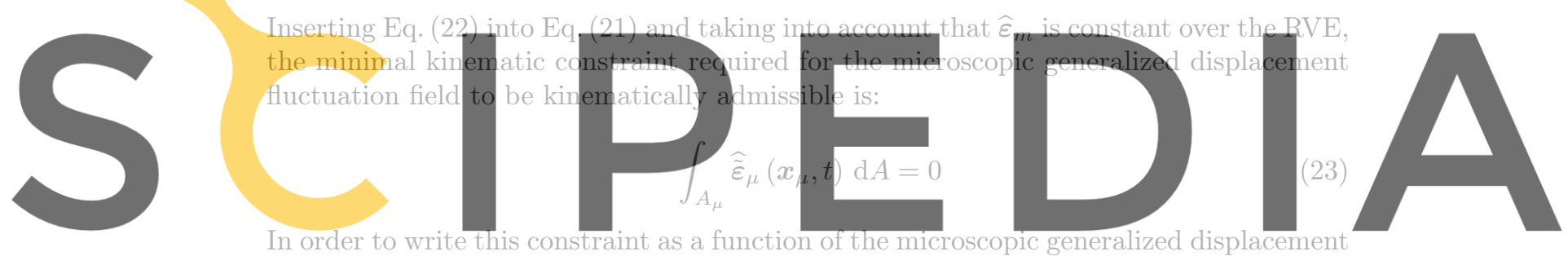

fluctuation field, it is useful to split Eq. (23) into membrane $\tilde{\varepsilon}_{0, \mu}$, bending $\tilde{\kappa}_{\mu}$, and shear

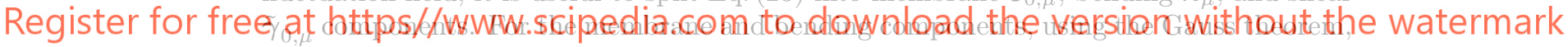

the surface integrals can be rewritten as boundary integrals over the RVE boundary $\partial A_{\mu}$ :

$$
\begin{gathered}
\int_{A_{\mu}} \tilde{\boldsymbol{\varepsilon}}_{0, \mu} \mathrm{d} A=\int_{A_{\mu}} \nabla^{s} \tilde{\boldsymbol{u}}_{0, \mu} \mathrm{d} A=\int_{\partial A_{\mu}} \tilde{\boldsymbol{u}}_{0, \mu} \otimes_{s} \boldsymbol{n} \mathrm{d} S=0 \\
\int_{A_{\mu}} \tilde{\boldsymbol{\kappa}}_{\mu} \mathrm{d} A=\int_{A_{\mu}} \nabla^{s}\left(\boldsymbol{P} \tilde{\boldsymbol{\theta}}_{\mu}\right) \mathrm{d} A=\int_{\partial A_{\mu}}\left(\boldsymbol{P} \tilde{\boldsymbol{\theta}}_{\mu}\right) \otimes_{s} \boldsymbol{n} \mathrm{d} S=0
\end{gathered}
$$

Eq. (24) and Eq. (25) provide the minimal kinematic constraint to be applied to the micro in-plane displacement fluctuations $\tilde{\boldsymbol{u}}_{0, \mu}$ and to the micro out-of-plane rotation fluctuations $\tilde{\boldsymbol{\theta}}_{\mu}$.

As regarding the shear component, the following minimal constraint has to be satisfied:

$$
\int_{A_{\mu}} \tilde{\gamma}_{0, \mu} \mathrm{d} A=\int_{A_{\mu}} \nabla \tilde{u}_{z, \mu}+\boldsymbol{P} \tilde{\boldsymbol{\theta}}_{\mu} \mathrm{d} A=0
$$

This constraint can be either imposed directly as a surface integral, or, if periodic conditions for the out-of-plane displacement fluctuation $\tilde{u}_{z}$ are to be used, only the following constraint should be applied as a surface integral over the RVE domain: 


$$
\int_{A_{\mu}} \tilde{\boldsymbol{\theta}}_{\mu} \mathrm{d} A=0
$$

The previously defined minimal conditions can be imposed using different kinds of boundary conditions. In the present work, taking advantage of the periodic nature of the considered micro-structure, the following set of constraints have been adopted:

$$
\begin{gathered}
\tilde{\boldsymbol{u}}_{0, \mu}\left(\boldsymbol{x}_{\mu}^{+}, t\right)=\tilde{\boldsymbol{u}}_{0, \mu}\left(\boldsymbol{x}_{\mu}^{-}, t\right) \quad \forall \text { pair }\left\{\boldsymbol{x}_{\mu}^{+}, \boldsymbol{x}_{\mu}^{-}\right\} \in \partial A_{\mu} \\
\tilde{\boldsymbol{\theta}}_{\mu}\left(\boldsymbol{x}_{\mu}^{+}, t\right)=\tilde{\boldsymbol{\theta}}_{\mu}\left(\boldsymbol{x}_{\mu}^{-}, t\right) \quad \forall \text { pair }\left\{\boldsymbol{x}_{\mu}^{+}, \boldsymbol{x}_{\mu}^{-}\right\} \in \partial A_{\mu} \\
\tilde{u}_{z, \mu}\left(x_{\mu}^{+}, t\right)=\tilde{u}_{z, \mu}\left(x_{\mu}^{-}, t\right) \quad \forall \operatorname{pair}\left\{x_{\mu}^{+}, x_{\mu}^{-}\right\} \in \partial A_{\mu} \\
\int_{A_{\mu}} \tilde{\theta}_{\mu}\left(x_{\mu}, t\right) \mathrm{d} A=0
\end{gathered}
$$

Eq. (28), Eq. (29) and Eq. (30) impose periodicity on the microscopic fluctuation fields.

This assumption showed a very good performance in a previous work by the authors on
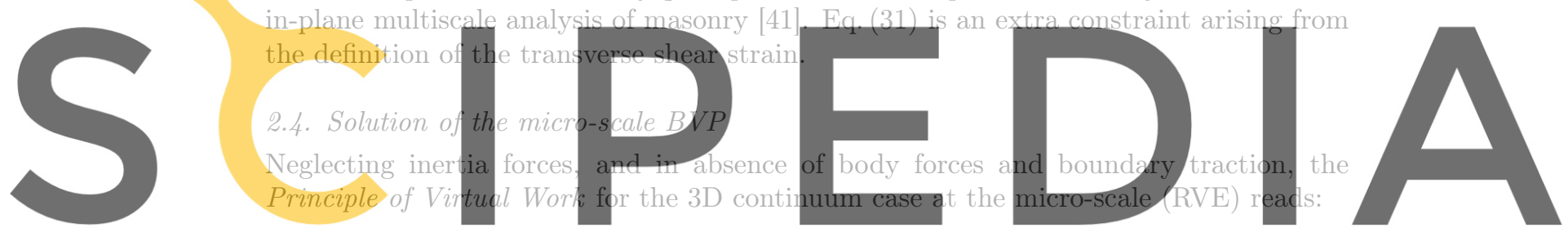

Register for free at https//www.scipedia.dom to download the version without the watermark

Considering that the shell works with generalized strains and stresses obtained from a through-the-thickness integration, Eq. (32) can be rewritten in terms of these generalized quantities:

$$
\begin{gathered}
\int_{A_{\mu}}\left\{\int_{-\frac{H}{2}}^{\frac{H}{2}} \boldsymbol{\sigma}_{\mu}(\zeta): \delta \tilde{\varepsilon}_{\mu}(\zeta) \mathrm{d} \zeta\right\} \mathrm{d} A=0 \\
\int_{A_{\mu}}\left\{\int_{-\frac{H}{2}}^{\frac{H}{2}} \boldsymbol{\sigma}_{\mu}(\zeta):\left(\delta \tilde{\varepsilon}_{0, \mu}+\zeta \delta \tilde{\boldsymbol{\kappa}}_{\mu}+\delta \tilde{\boldsymbol{\gamma}}_{0, \mu}\right) \mathrm{d} \zeta\right\} \mathrm{d} A=0 \\
\int_{A_{\mu}}\left\{\int _ { - \frac { H } { 2 } } ^ { \frac { H } { 2 } } \left[\sigma_{\mu, x x}\left(\delta \tilde{\varepsilon}_{0, \mu, x x}+\zeta \delta \tilde{\kappa}_{\mu, x x}\right)+\sigma_{\mu, y y}\left(\delta \tilde{\varepsilon}_{0, \mu, y y}+\zeta \delta \tilde{\kappa}_{\mu, y y}\right)+\right.\right. \\
\sigma_{\mu, x y}\left(2 \delta \tilde{\varepsilon}_{0, \mu, x y}+2 \zeta \delta \tilde{\kappa}_{\mu, x y}\right)+ \\
\left.\left.\sigma_{\mu, x z}\left(2 \delta \tilde{\varepsilon}_{0, \mu, x z}\right)+\sigma_{\mu, y z}\left(2 \delta \tilde{\varepsilon}_{0, \mu, y z}\right)\right] \mathrm{d} \zeta\right\} \mathrm{d} A=0
\end{gathered}
$$




$$
\begin{gathered}
\int_{A_{\mu}}\left[\boldsymbol{N}_{\mu}: \delta \tilde{\boldsymbol{\varepsilon}}_{0, \mu}+\boldsymbol{M}_{\mu}: \delta \tilde{\boldsymbol{\kappa}}_{\mu}+\boldsymbol{Q}_{\mu}: \delta \tilde{\boldsymbol{\gamma}}_{0, \mu}\right] \mathrm{d} A=0 \\
\int_{A_{\mu}} \widehat{\boldsymbol{\sigma}}_{\mu}: \delta \widehat{\tilde{\boldsymbol{\varepsilon}}}_{\mu} \mathrm{d} A=0
\end{gathered}
$$

\subsection{Up-scaling or micro-macro transition}

Upon RVE equilibrium, the homogenized generalized stress should be computed. As discussed in [20,31], the definition of the homogenized stress can be directly obtained from the Hill-Mandel Principle [50, 51] using the additive split of the microscopic strain. The Hill-Mandel Principle can be written in terms of generalized stresses and strains as:

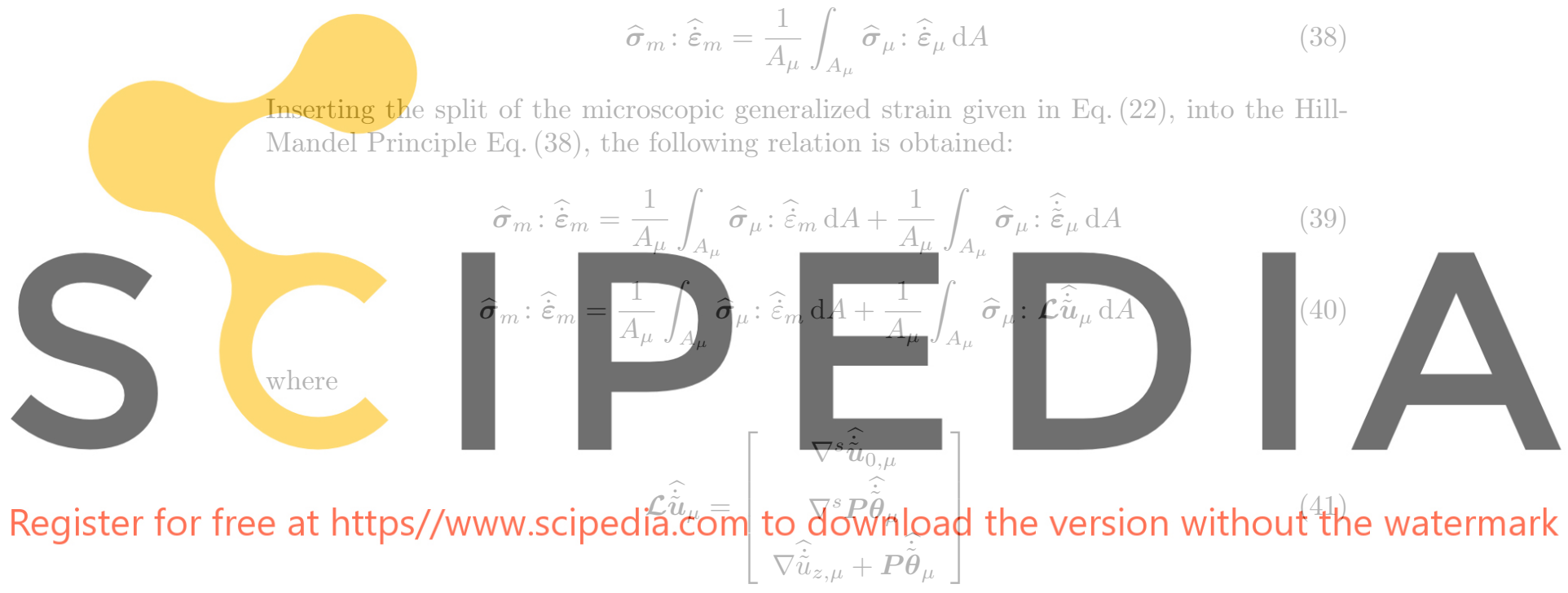

Eq. (40) is valid for any kinematically admissible $\widehat{\tilde{\tilde{\boldsymbol{u}}}}_{\mu}$. Specifically, in the case of $\widehat{\tilde{\boldsymbol{u}}}_{\mu}=0$, the following relation is obtained:

$$
\widehat{\boldsymbol{\sigma}}_{m}: \widehat{\dot{\varepsilon}}_{m}=\frac{1}{A_{\mu}} \int_{A_{\mu}} \widehat{\boldsymbol{\sigma}}_{\mu}: \widehat{\dot{\varepsilon}}_{m} \mathrm{~d} A \quad \forall \dot{\varepsilon}_{m}
$$

which is valid for any macroscopic generalized strain rate field, leading to the definition of the homogenized macroscopic generalized stress as the surface average of the microscopic generalized stress field:

$$
\widehat{\boldsymbol{\sigma}}_{m}=\frac{1}{A_{\mu}} \int_{A_{\mu}} \widehat{\boldsymbol{\sigma}}_{\mu} \mathrm{d} A
$$

From a computational point of view, it is more convenient to obtain the homogenized generalized stresses from the reactions obtained on the RVE boundaries upon equilibrium [44]: 


$$
\begin{gathered}
\boldsymbol{N}=\frac{1}{A_{\mu}} \sum_{i=1}^{n} \boldsymbol{f}_{r, i} \otimes^{s} \boldsymbol{x}_{\mu, i} \\
\boldsymbol{M}=\frac{1}{A_{\mu}} \boldsymbol{P} \sum_{i=1}^{n} \boldsymbol{m}_{r, i} \otimes^{s} \boldsymbol{x}_{\mu, i}-\frac{1}{A_{\mu}} \sum_{i=1}^{n} f_{r z, i} \boldsymbol{x}_{\mu, i} \otimes \boldsymbol{x}_{\mu, i} \\
\boldsymbol{Q}=\frac{1}{A_{\mu}} \sum_{i=1}^{n} f_{r z, i} \boldsymbol{x}_{\mu, i}
\end{gathered}
$$

where $i=1,2, \ldots, n$ is the boundary node index, $\boldsymbol{x}_{\mu, i}=\left[\begin{array}{ll}x_{\mu, i} & y_{\mu, i}\end{array}\right]^{T}$ is the position vector of the boundary node $i, \boldsymbol{f}_{r, i}=\left[\begin{array}{ll}f_{r x, i} & f_{r y, i}\end{array}\right]^{T}$ is the in-plane reaction force vector, $\boldsymbol{m}_{r, i}=\left[\begin{array}{ll}m_{r x, i} & m_{r y, i}\end{array}\right]^{T}$ is the out-of-plane reaction moment vector, and $f_{r z, i}$ is the out-of-plane reaction force. The transformation matrix $\boldsymbol{P}$ in Eq. (45), is the same introduced in Eq. (6). The explicit expression for each homogenized macro generalized stress component is:

$$
\widehat{\boldsymbol{\sigma}}_{m}=\left[\begin{array}{c}
N_{x x} \\
N_{y y} \\
N_{x y} \\
M_{x x} \\
M_{y y} \\
M_{x y} \\
Q_{x z} \\
Q_{y z}
\end{array}\right]=\frac{1}{A_{\mu}} \sum_{i=1}^{n}\left[\begin{array}{c}
f_{r x, i} x_{\mu, i} \\
f_{r y, i} y_{\mu, i} \\
\frac{1}{2}\left(f_{r x, i} y_{\mu, i}+f_{r y, i} x_{\mu, i}\right) \\
m_{r y, i} x_{\mu, i}-\frac{1}{2} f_{r z, i} x_{\mu, i}^{2} \\
-m_{r x, i} y_{\mu, i}-\frac{1}{2} f_{r z, i} y_{\mu, i}^{2} \\
\frac{1}{2}\left(m_{r y, i} y_{\mu, i}-m_{r x, i} x_{\mu, i}\right)-\frac{1}{2} f_{r z, i} x_{\mu, i} y_{\mu, i} \\
f_{r z, i} x_{\mu, i} \\
f_{r z, i} y_{\mu, i}
\end{array}\right]
$$

\subsection{Typical deformation modes obtained from the shell homogenization framework}

This section shows the eight natural deformation modes obtained from the proposed homogenization framework for thick shells in terms of coarse scale displacements/rotations, fine scale displacement/rotation fluctuations, and total displacements/rotations. In the proposed method, the unknowns at the micro-scale are the fluctuations, so the total displacements/rotations are just computed and displayed as a post-process for visualization purposes.

The total microscopic displacement/rotation vector is defined as the sum of the coarse scale contribution and the fine scale contribution (fluctuations):

$$
\begin{gathered}
\widehat{\boldsymbol{u}}_{\mu}=\widehat{\boldsymbol{u}}_{m}+\widehat{\tilde{\boldsymbol{u}}}_{\mu} \\
{\left[\begin{array}{lll}
\boldsymbol{u}_{0, \mu} & u_{z, \mu} & \boldsymbol{\theta}_{\mu}
\end{array}\right]^{T}=\left[\begin{array}{lll}
\boldsymbol{u}_{0, m} & u_{z, m} & \boldsymbol{\theta}_{m}
\end{array}\right]^{T}+\left[\begin{array}{lll}
\tilde{\boldsymbol{u}}_{0, \mu} & \tilde{u}_{z, \mu} & \tilde{\boldsymbol{\theta}}_{\mu}
\end{array}\right]^{T}}
\end{gathered}
$$

The microscopic displacement/rotation fluctuations $\widehat{\tilde{\boldsymbol{u}}}_{\mu}$ are obtained by the solution of the microscopic boundary value problem, while the displacement/rotation vector due to 
the coarse scale contribution $\widehat{\boldsymbol{u}}_{m}$ can be calculated as a post-process from the macroscopic generalized strains $\widehat{\varepsilon}_{m}$ :

$$
\begin{gathered}
\boldsymbol{u}_{0, m}=\boldsymbol{\varepsilon}_{0, m} \boldsymbol{x}_{\mu} \\
\boldsymbol{\theta}_{m}=\boldsymbol{P}^{T} \boldsymbol{\kappa}_{m} \boldsymbol{x}_{\mu} \\
u_{z, m}=-\frac{1}{2}\left(\boldsymbol{\kappa}_{m} \boldsymbol{x}_{\mu}\right) \cdot \boldsymbol{x}_{\mu}+\boldsymbol{\gamma}_{m} \cdot \boldsymbol{x}_{\mu}
\end{gathered}
$$

In Eq. (51), the transformation matrix $\boldsymbol{P}$ is still the one defined in Eq. (6) to take into account the sign convention of rotations. In Eq. (52), the first term represents the deflection due to the bending deformation, while the second term represents the deflection due to the shear deformation. Figure 2 to Figure 9 show all the eight deformation modes associated with the eight deformation components of the macroscopic generalized strain vector. In all of them, (a) shows the displacement/rotation field $\widehat{\boldsymbol{u}}_{m}$ arising from the associated average macroscopic deformation mode representing the coarse-scale contribution, (b) shows the microscopic displacement/rotation fluctuation field $\widehat{\tilde{\boldsymbol{u}}}_{\mu}$ (unknown of the microscopic BVP) that represents the fine-scale contribution accounting for the heterogeneity (bricks and mortar), and (c) represents the total microscopic displacement/rotation $\widehat{\boldsymbol{u}}_{\mu}$ as the superposition of coarse and fine scale contributions. Figure 2, Figure 3 and Figure 4 show the three membrane deformation modes. Figure 5, Figure 6 and Figure 7 show the three bending deformation modes. Figure 8 and Figure 9 show the two transverse shear deformation modes.

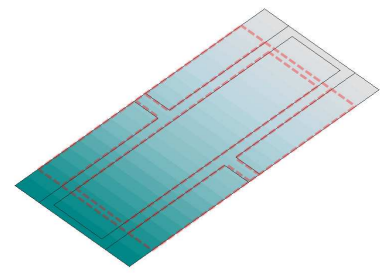

(a)

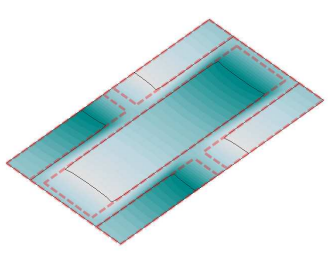

(b)

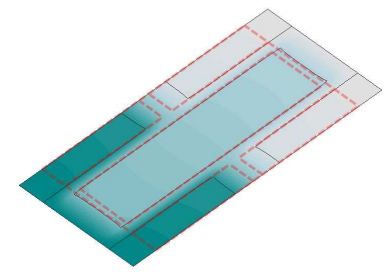

(c)

Figure 2: deformation mode due to $\varepsilon_{x x}$. (a) coarse scale, (b) fine scale, (c) total 


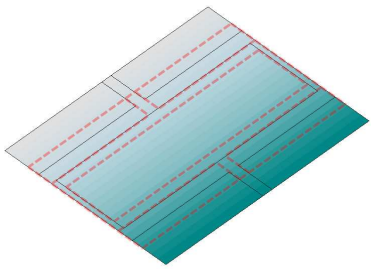

(a)

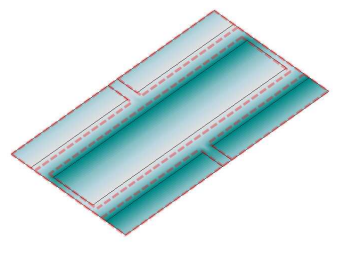

(b)

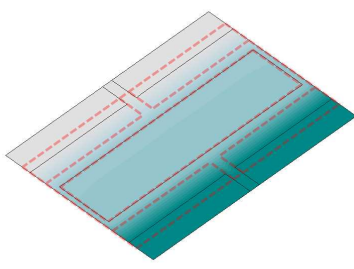

(c)

Figure 3: deformation mode due to $\varepsilon_{y y}$. (a) coarse scale, (b) fine scale, (c) total

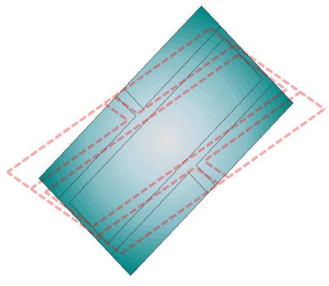

(a)

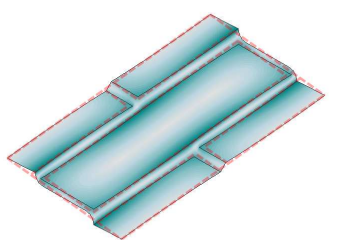

(b)

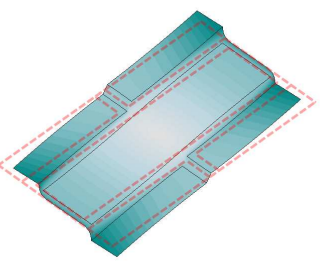

(c)

Figure 4: deformation mode due to $\varepsilon_{x y}$. (a) coarse scale, (b) fine scale, (c) total

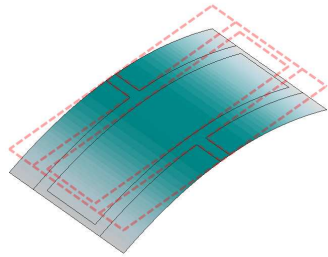

(a)

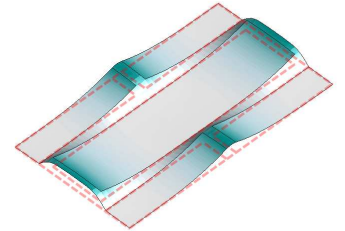

(b)

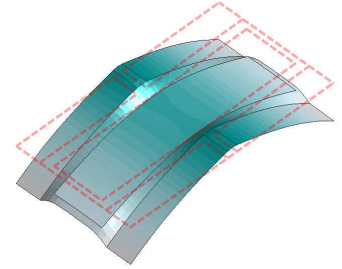

(c)

Figure 5: deformation mode due to $\kappa_{x x}$. (a) coarse scale, (b) fine scale, (c) total 


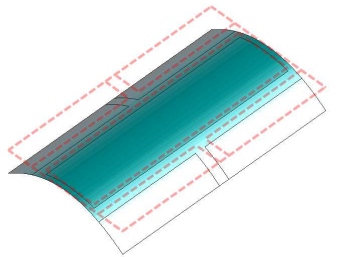

(a)

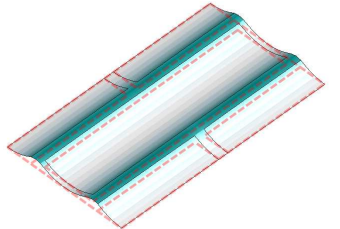

(b)

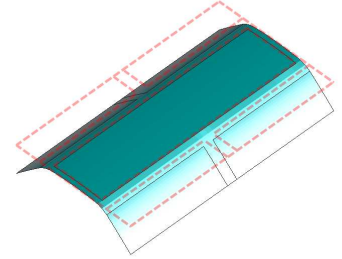

(c)

Figure 6: deformation mode due to $\kappa_{y y}$. (a) coarse scale, (b) fine scale, (c) total

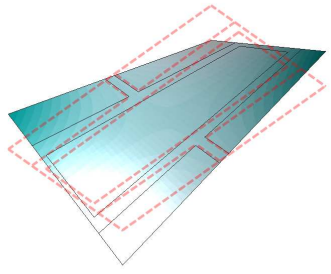

(a)

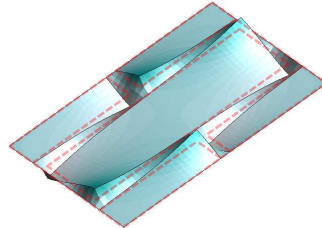

(b)

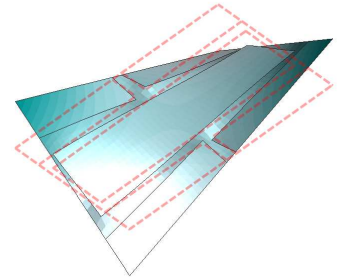

(c)

Figure 7: deformation mode due to $\kappa_{x y}$. (a) coarse scale, (b) fine scale, (c) total

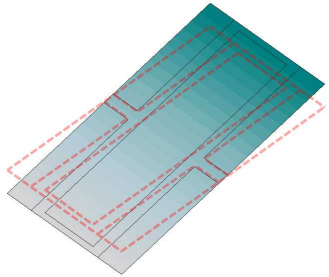

(a)

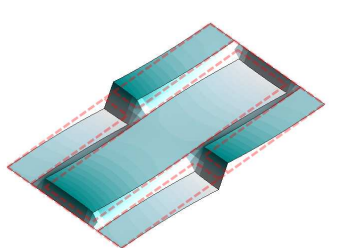

(b)

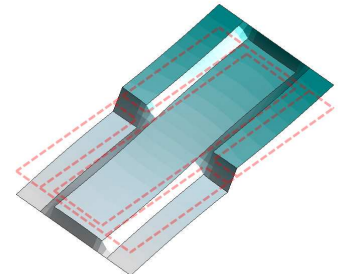

(c)

Figure 8: deformation mode due to $\gamma_{x z}$. (a) coarse scale, (b) fine scale, (c) total 


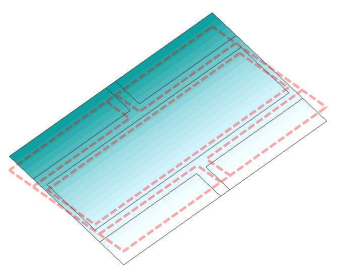

(a)

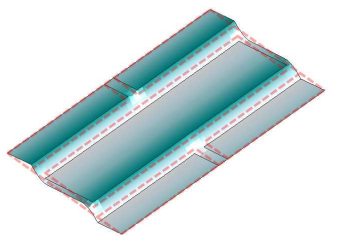

(b)

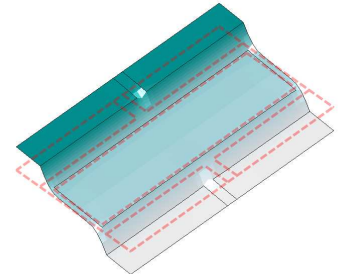

(c)

Figure 9: deformation mode due to $\gamma_{y z}$. (a) coarse scale, (b) fine scale, (c) total

\section{Fracture-energy regularization in 2-scale FE computational homogeniza- tion}

A generalized geometrical characteristic length, accounting for the size of (i) macro-scale FE, (ii) micro-scale FE and (iii) RVE, was proposed in a previous work by the authors [41]. This fracture-energy regularization for 2-scale FE analysis ensures that the energy dissipated at the macro-scale be objective with respect to the discretization size in both micro and macro scales, and with respect to the size of the RVE. Only a summary is given here, while the reader can refer to [41] for further details.

Micro-scale local constitutive models are regularized with a modified characteristic length $\widetilde{l}_{c h, \mu}$, which depends on all the characteristic lengths involved in the homogenization problem (see Figure 10):

$$
\widetilde{l}_{c h, \mu}=f\left(l_{c h, \mu}, l_{c h, m}, l_{c h, R V E}\right)
$$

where $l_{c h, \mu}, l_{c h, m}$ and $l_{c h, R V E}$ are respectively the micro-scale FE characteristic length, the macro-scale FE characteristic length, and the RVE characteristic length. $W_{R V E_{D}}$ is the energy dissipated in the damaged domain $\left(V_{R V E,_{D}}\right)$ of the RVE:

$$
W_{R V E, D}=\frac{G_{f}}{\widetilde{l}_{c h, \mu}} V_{R V E,_{D}}=\frac{G_{f}}{\widetilde{l}_{c h, \mu}} l_{c h, \mu} t_{\mu} h_{R V E}
$$

whit $h_{R V E}$ being the length of the RVE in the direction of the crack, and $t_{\mu}$ being the thickness of the RVE. "Smearing" this energy over the entire RVE domain $\left(V_{R V E}\right)$, an equivalent specific fracture energy $\widetilde{g}_{f}$ is obtained:

$$
\widetilde{g}_{f}=\frac{W_{R V E_{, D}}}{V_{R V E}}=\frac{W_{R V E_{, D}}}{l_{c h, R V E} h_{R V E} t_{\mu}}=\frac{G_{f}}{\widetilde{l}_{c h, \mu}} \frac{l_{c h, \mu}}{l_{c h, R V E}}
$$

$\widetilde{g}_{f}$ can be integrated over the dissipating macro-scale domain to give the total energy $W_{m}$ dissipated at the macro-scale. Equating $W_{m}$ to the actual dissipated energy $W$ 


$$
\begin{gathered}
W_{m}=W \\
\widetilde{g}_{f} l_{c h, m} h_{m} t_{m}=G_{f} h_{m} t_{m}
\end{gathered}
$$

gives a definition for the modified micro-scale FE characteristic length:

$$
\widetilde{l}_{c h, \mu}=l_{c h, \mu} \frac{l_{c h, m}}{l_{c h, R V E}}
$$

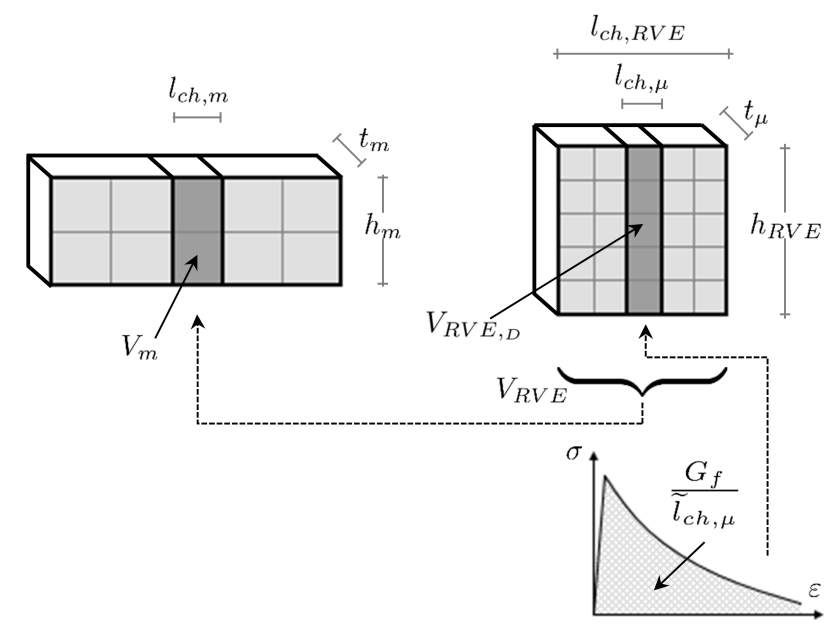

Figure 10: fracture energy based regularization in 2-scale FEM [41]

It should be noticed that this regularization procedure is an extension of the classical crack band theory, adapted to the 2 -scale homogenization problem. As such, it regularizes globally the problem, but still considers local values in the localization zone.

\section{Numerical modeling of out-of-plane loaded masonry walls}

The proposed method is applied to the simulation of the out-of-plane behavior of brickmasonry walls. Experimental tests on Unreinforced Masonry Walls (URM) presented in [47] are considered here for the numerical assessment of the method. Section 4.1 briefly describes the experimental test. The nonlinear behavior of bricks and mortar joints is modeled with a constitutive model based on Continuum-Damage Mechanics, described in Section 4.2, while the adopted RVE and material parameters are described in Section 4.3. A preliminary Direct Numerical Simulation (DNS) was performed in order to assess the capability of the constitutive model to emulate the specific local responses of the masonry wall under out-of-plane loadings, and the results are discussed in Section 4.4. Multiscale simulations are discussed in Section 4.5, and the results are compared with both the experimental test and the DNS. 
The analyses were carried out using Kratos Multiphysics [52, 53], a free open-source framework for the development of multidisciplinary solvers, developed at CIMNE. Pre and post-processing were carried out in GiD [54], also developed at CIMNE.

\subsection{Experimental test}

Experimental tests on Unreinforced Masonry Walls (URM) have been presented in [47]. Eight full-scale clay brick URM walls were subjected to quasi-static displacement-controlled loading to investigate their load-deflection behavior beyond their point of maximum strength. Of the eight walls analyzed in the experimental campaign, only one is considered here. The wall measures $4 \mathrm{~m} \times 2.5 \mathrm{~m}$, with bricks of $230 \mathrm{~mm} \times 76 \mathrm{~mm} \times 110 \mathrm{~mm}$, and mortar joints of $10 \mathrm{~mm}$. After the first stage where a vertical pre-compression of $0.1 \mathrm{~N} / \mathrm{mm}^{2}$ is applied, the wall is subjected to a uniform pressure under displacement control. In order to provide a realistic full moment connection along the vertical edges, $450 \mathrm{~mm}$ long return walls were built in as part of the tested wall. Figure 11 shows the wall geometry and the obtained failure pattern on the main face of the wall, and on the lateral turning walls.
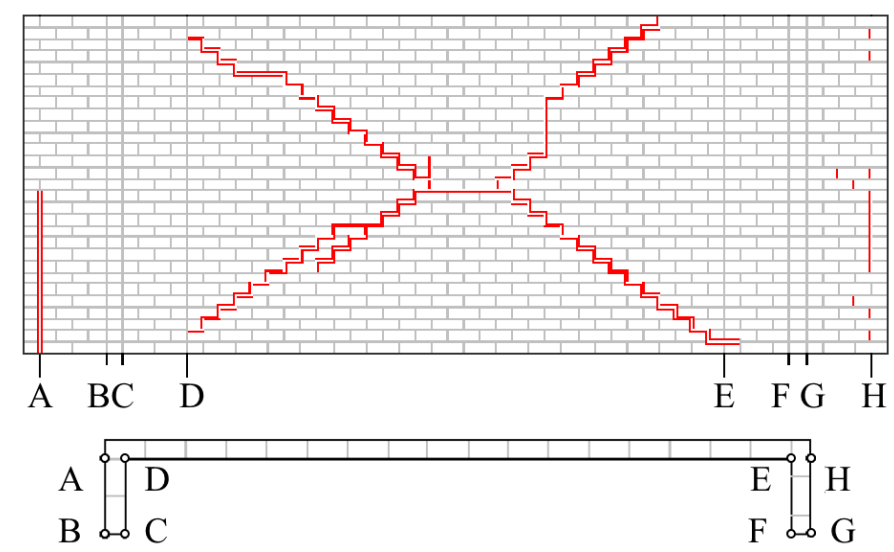

Figure 11: Tested wall geometry and obtained failure pattern [47]

\subsection{Tension/Compression continuum damage model}

\subsubsection{Constitutive Model}

The bi-dissipative $d^{+} / d^{-}$damage model, based on the works in $[55,56,57]$, defines the stress tensor as

$$
\boldsymbol{\sigma}=\left(1-d^{+}\right) \overline{\boldsymbol{\sigma}}^{+}+\left(1-d^{-}\right) \overline{\boldsymbol{\sigma}}^{-}
$$

with $\overline{\boldsymbol{\sigma}}^{+}$and $\overline{\boldsymbol{\sigma}}^{-}$being respectively the positive and negative parts of the effective (elastic) stress tensor $\overline{\boldsymbol{\sigma}}$ : 


$$
\overline{\boldsymbol{\sigma}}=\boldsymbol{C}: \varepsilon, \quad \overline{\boldsymbol{\sigma}}^{+}=\sum_{i=1}^{3}\left\langle\bar{\sigma}_{i}\right\rangle \boldsymbol{p}_{i} \otimes \boldsymbol{p}_{i}, \quad \overline{\boldsymbol{\sigma}}^{-}=\overline{\boldsymbol{\sigma}}-\overline{\boldsymbol{\sigma}}^{+}
$$

$d^{+}$and $d^{-}$are, respectively, the tensile and compressive damage indexes, acting respectively on $\overline{\boldsymbol{\sigma}}^{+}$and $\overline{\boldsymbol{\sigma}}^{-}$. The damage indexes are scalar variables ranging from 0 (intact material) to 1 (completely damaged material).

\subsubsection{Failure Criteria}

In order to identify "loading", "unloading" or "reloading" conditions of a general state of stress, two scalar measures are introduced, termed as equivalent stresses $\tau^{+}$and $\tau^{-}$. Both compressive and tensile failure criteria are based on the one described in [58], and are computed as:

$$
\begin{gathered}
\tau^{-}=H\left(-\bar{\sigma}_{\text {min }}\right)\left[\frac{1}{1-\alpha}\left(\alpha \bar{I}_{1}+\sqrt{3 \bar{J}_{2}}+k_{1} \beta\left\langle\bar{\sigma}_{\text {max }}\right\rangle+\gamma\left\langle-\bar{\sigma}_{\text {max }}\right\rangle\right)\right] \\
\tau^{+}=H\left(\bar{\sigma}_{\text {max }}\right)\left[\frac{1}{1-\alpha}\left(\alpha \bar{I}_{1}+\sqrt{3 \bar{J}_{2}}+\beta\left\langle\bar{\sigma}_{\text {max }}\right\rangle\right) \frac{f_{t}}{f_{c p}}\right]
\end{gathered}
$$

with

$$
\begin{gathered}
\alpha=\frac{k_{b}-1}{2 k_{b}-1} \\
\beta=\frac{f_{c p}}{f_{t}}(1-\alpha)-(1+\alpha)
\end{gathered}
$$

where $\bar{I}_{1}$ is the first invariant of the effective stress tensor, $\bar{J}_{2}$ is the second invariant of the effective deviatoric stress tensor, $\bar{\sigma}_{\max }$ is the maximum effective principal stress, $f_{c p}$ is the compressive peak stress, $k_{b}$ is the ratio of bi-axial to uniaxial compressive strengths, and the term $\frac{f_{t}}{f_{c p}}$ is used to relate $\tau^{+}$to the tensile strength $f_{t}$. The constant $k_{1}$ in Eq. (60) controls shear behavior of the model acting on the shape of the compressive surface. $k_{1}$ can range from 0 (Drucker-Prager criterion) to 1 (criterion proposed in [58]). Finally the last term in Eq. (60), with a $\gamma>0$, accounts for increasing strength under stress states of triaxial compression. The two damage surfaces for the $2 \mathrm{D}$ plane-stress case are shown in Figure 12, while the same surfaces for the 3D case are shown in Figure 13. 


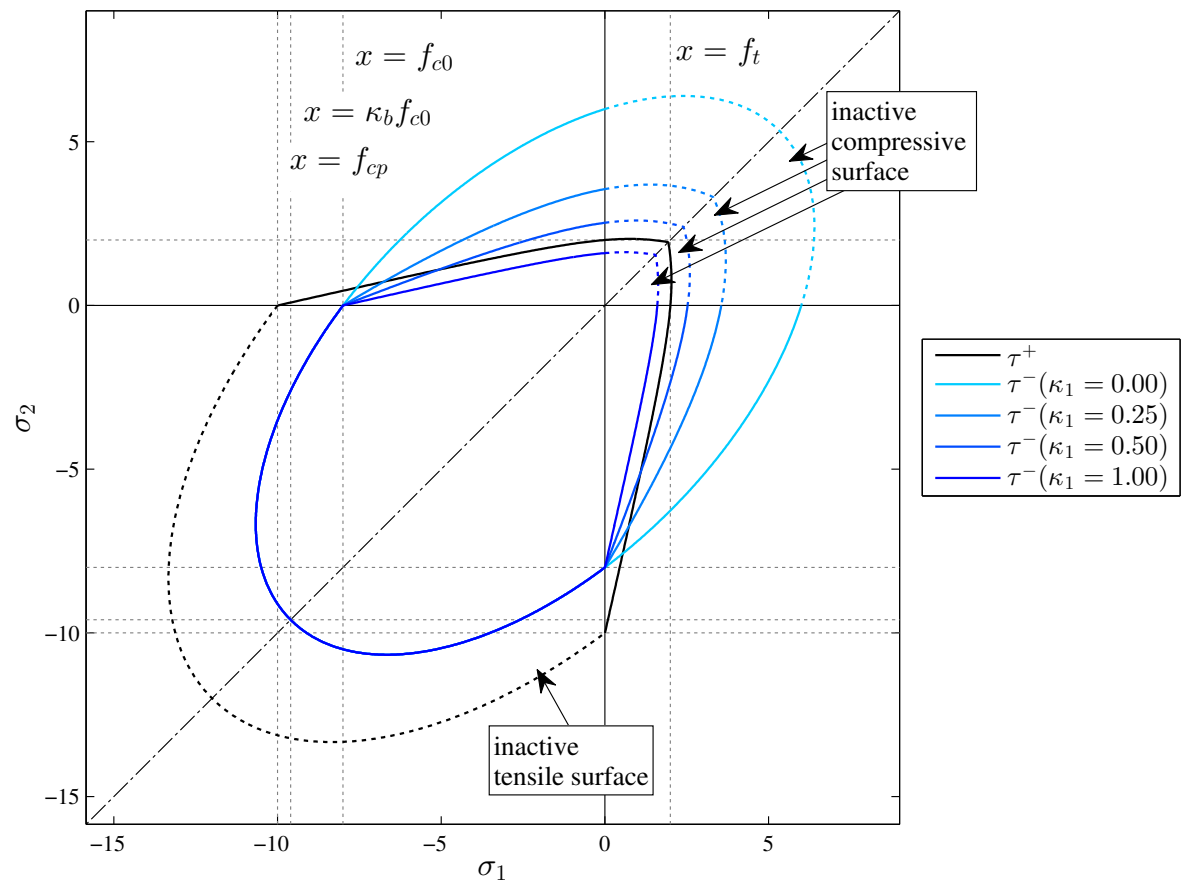

Figure 12: initial damage surfaces (2D plane-stress)

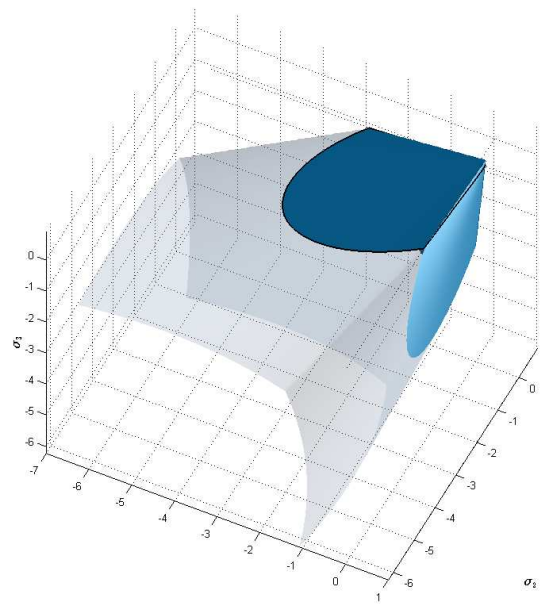

(a) tensile surface

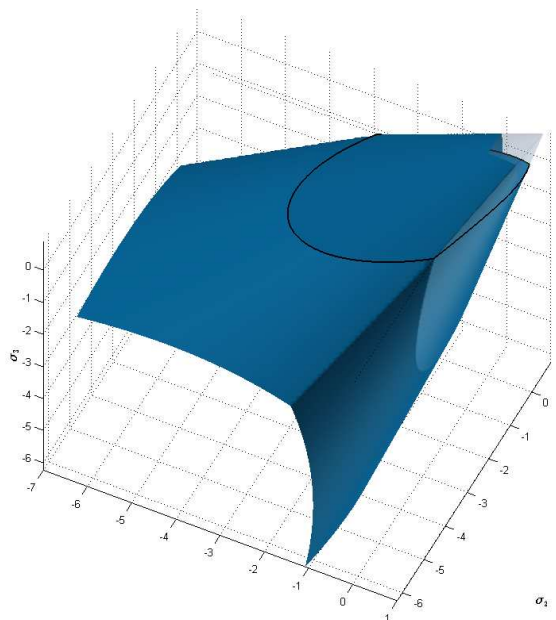

(b) compressive surface

Figure 13: initial damage surfaces (3D). Inactive surfaces in transparency 
Note that both compressive and tensile surfaces are defined for any stress state, it is therefore necessary to inactivate them under certain conditions:

1. the compressive surface can evolve if and only if at least one principal stress is negative

2. the tensile surface can evolve if and only if at least one principal stress is positive

These conditions are achieved with the Heaviside function $H(x)$ introduced in Eq. (60) and Eq. (61). The inactive portions of the two surfaces, i.e. those portions cut out by the Heaviside function, are drawn with dashed-lines in Figure 12, and in transparency in Figure 13.

To represent the irreversible nature of the damage process, other two scalar quantities, termed as damage thresholds $r^{ \pm}$, are introduced. They represent the largest values attained by the equivalent stresses $\tau^{ \pm}$during the loading history. Therefore $r^{ \pm}$at time $t+\Delta t$ can be explicitly evaluated as

$$
r^{ \pm}=\max \left(r_{0}^{ \pm}, \max _{0 \leq n \leq t} \tau_{n}^{ \pm}\right), \quad r_{0}^{+}=f_{t}, \quad r_{0}^{-}=f_{c 0}
$$

where $r_{0}^{+}$and $r_{0}^{-}$are, respectively, the initial tensile and compressive damage thresholds (i.e. the elastic limits in uniaxial tension $f_{t}$ and compression $f_{c 0}$ ), and $n$ denotes the time instant. Accordingly, the following damage criteria are defined:

$$
\Phi\left(\tau^{ \pm}, r^{ \pm}\right)=\tau^{ \pm}-r^{ \pm} \leq 0
$$

\subsubsection{Evolution laws for damage variables}

The tensile damage $d^{+}$is obtained with the following exponential softening law:

$$
d^{+}\left(r^{+}\right)=1-\frac{r_{0}^{+}}{r^{+}} \exp \left\{2 H_{\text {dis }}\left(\frac{r_{0}^{+}-r^{+}}{r_{0}^{+}}\right)\right\}
$$

where $H_{d i s}$ is the discrete softening parameter. In the discrete problem, when using local constitutive models, the softening law should be adjusted depending on the width of the dissipative zone $\left(l_{d i s}\right)[59,60,61]$, so that the following equality holds:

$$
g_{f} l_{d i s}=G_{f}
$$

where the specific dissipated energy $g_{f}$ is

$$
g_{f}=\left(1+\frac{1}{H_{\text {dis }}}\right) \frac{f_{t}^{2}}{2 E}
$$

$H_{d i s}$ is calculated as

$$
H_{d i s}=\frac{l_{d i s}}{l_{m a t}-l_{d i s}}
$$

with $l_{m a t}=2 E G_{f} / f_{t}^{2} . l_{d i s}$ is taken equal to the FE characteristic length $\left(l_{d i s}=l_{c h}\right)$. 


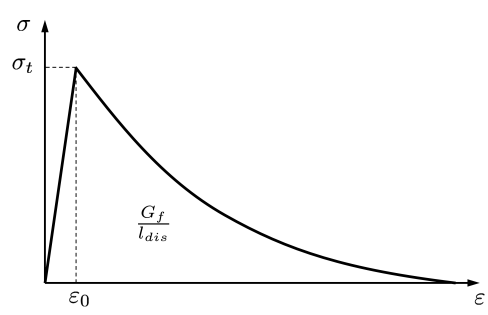

(a)

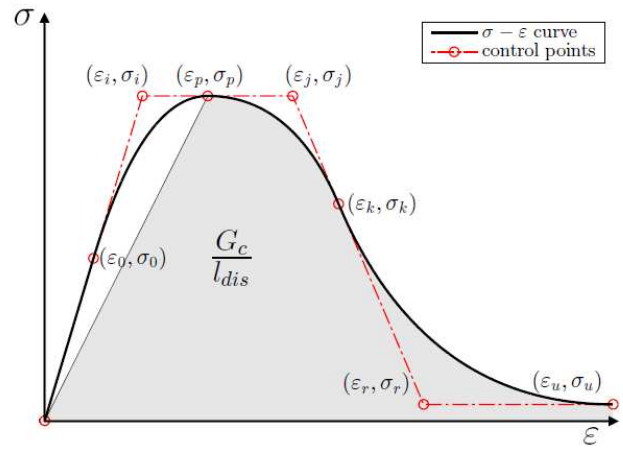

(b)

Figure 14: (a) tensile and (b) compressive uniaxial laws

The obtained tensile uniaxial law is shown in Figure 14a. The evolution of the compressive damage $d^{-}$is driven by a hardening/softening law (see Figure 14b) made of three quadratic Bezier curves, as described in a previous work by the authors [41]. As for the tensile case, in the discrete problem the compressive curve shown in Figure 14b needs to be regularized so that the shaded area underneath be $G_{c} / l_{d i s}$.

\subsection{RVE and material parameters}

The geometry of the adopted RVE is shown in Figure 15. The boundary conditions for the RVE are those listed from Eq. (28) to Eq. (31)

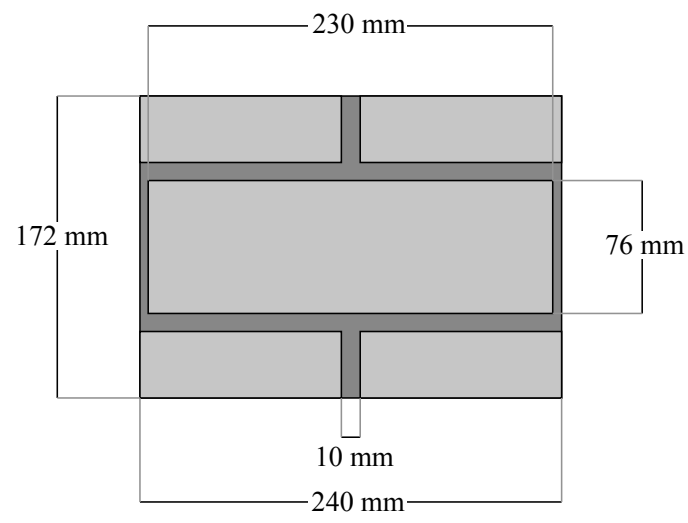

Figure 15: RVE geometry. 
The constitutive model used for both bricks and mortar joints is based on Continuum Damage Mechanics, and it is briefly described in Section 4.2.

Material properties for bricks and mortar are summarized in Table 1.

\begin{tabular}{cccccccccccc}
\hline$E$ & $\nu$ & $\sigma_{t}$ & $G_{t}$ & $\sigma_{0}$ & $\sigma_{p}$ & $\sigma_{r}$ & $G_{c}$ & $\varepsilon_{p}$ & $k_{b}$ & $k_{1}$ & $\gamma$ \\
\hline \hline 52700.0 & 0.15 & 2.0 & 0.08 & 9.0 & 18.0 & 2.5 & 40.0 & 0.003 & 1.2 & 0.0 & 3.0 \\
\hline$\frac{N}{m m^{2}}$ & - & $\frac{N}{m m^{2}}$ & $\frac{N}{m m}$ & $\frac{N}{m m^{2}}$ & $\frac{N}{m m^{2}}$ & $\frac{N}{m m^{2}}$ & $\frac{N}{m m}$ & - & - & - & - \\
\hline
\end{tabular}

(a)

\begin{tabular}{cccccccccccc}
\hline$E$ & $\nu$ & $\sigma_{t}$ & $G_{t}$ & $\sigma_{0}$ & $\sigma_{p}$ & $\sigma_{r}$ & $G_{c}$ & $\varepsilon_{p}$ & $k_{b}$ & $k_{1}$ & $\gamma$ \\
\hline \hline 1000.0 & 0.15 & 0.15 & 0.003 & 1.0 & 2.5 & 0.5 & 30.0 & 0.02 & 1.2 & 0.16 & 1.0 \\
\hline$\frac{N}{m m^{2}}$ & - & $\frac{N}{m m^{2}}$ & $\frac{N}{m m}$ & $\frac{N}{m m^{2}}$ & $\frac{N}{m m^{2}}$ & $\frac{N}{m m^{2}}$ & $\frac{N}{m m}$ & - & - & - & - \\
\hline
\end{tabular}

(b)

Table 1: Material properties for (a) bricks and (b) mortar joints

\subsection{Micro-modeling or direct numerical simulation}

In the DNS the entire micro-structure is discretized in the computational model with shell elements, taking advantage of the fact that the micro-structure is heterogeneous in the in-plane direction but (initially) homogeneous in the thickness direction (1-layer of bricks). The geometry of the micro-model is shown in Figure 16. Due to the symmetry of both geometry and boundary conditions, only the left-half of the wall has been modeled. Figure 17 shows the pressure-deflection curve obtained from the DNS and compared with the experimental results. The deflection is measured at the center of the wall, where the maximum value is attained. Overall the micro-model simulation shows a good agreement with the experiment. Figure 18 shows the deformed shape at the end of the analysis, with a good agreement in the prediction of the failure mode shown in Figure 11.

The most important feature shown by the experimental campaign, as reported in [47], is the "apparent" plastic behavior of the tested walls: after reaching the wall strength at deflections between 2.0 and $5.0 \mathrm{~mm}$, a first loss of bearing capacity is shown, soon followed by an almost constant strength plateau up to a deflection of $25.0 \mathrm{~mm}$. As discussed by the authors of the experimental campaign, this apparent plastic behavior may be attributed to a redistribution of bending moment along diagonal cracks to horizontal bending along the vertical edges, where the bending restraint provided by the return walls has additional capacity to accept transfer of load from the diagonal bending mechanism. Table 2 shows the evolution of tensile and compressive damage during the first stages of the analysis, where the wall strength is attained, and the damage pattern at the end of the simulation. 


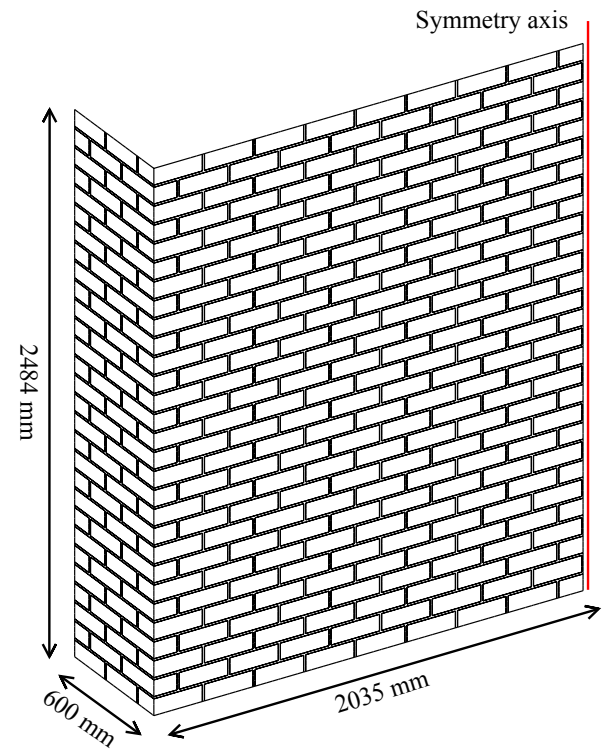

Figure 16: Micro-model geometry

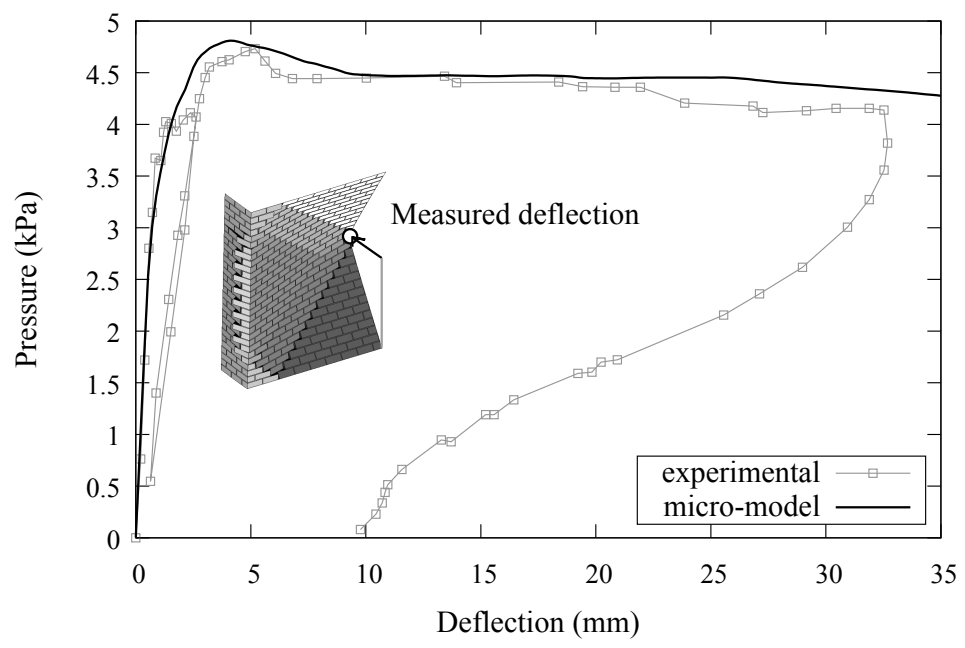

Figure 17: Pressure-deflection curve obtained from the micro-model analysis 


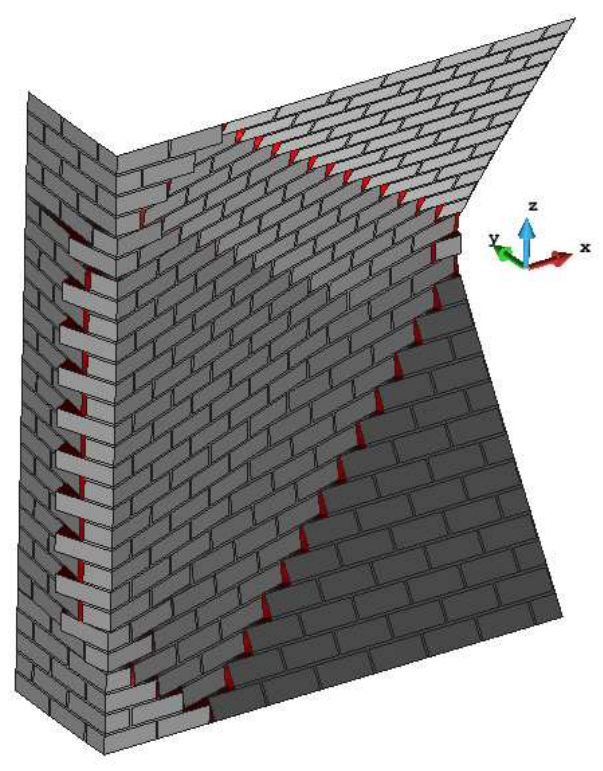

(a)

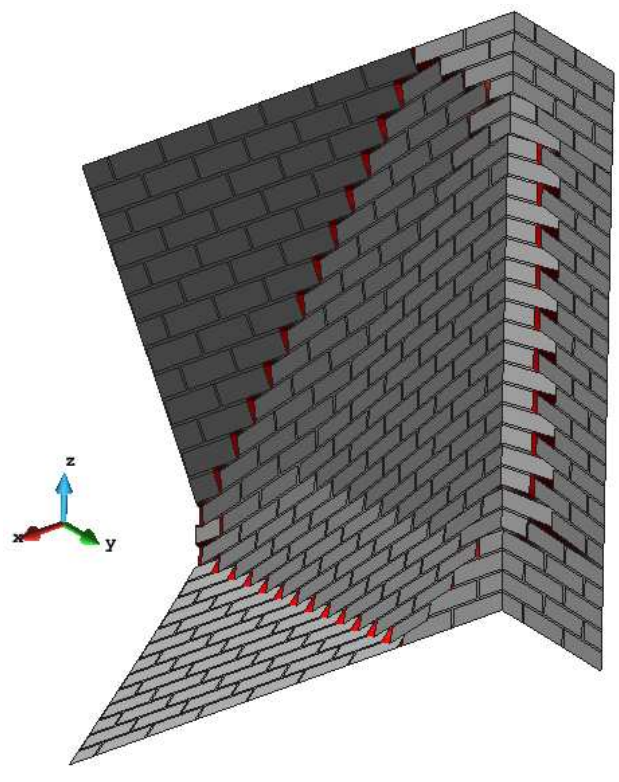

(b)

Figure 18: final deformed shape, (a) front and (b) back view from the micro-model analysis 


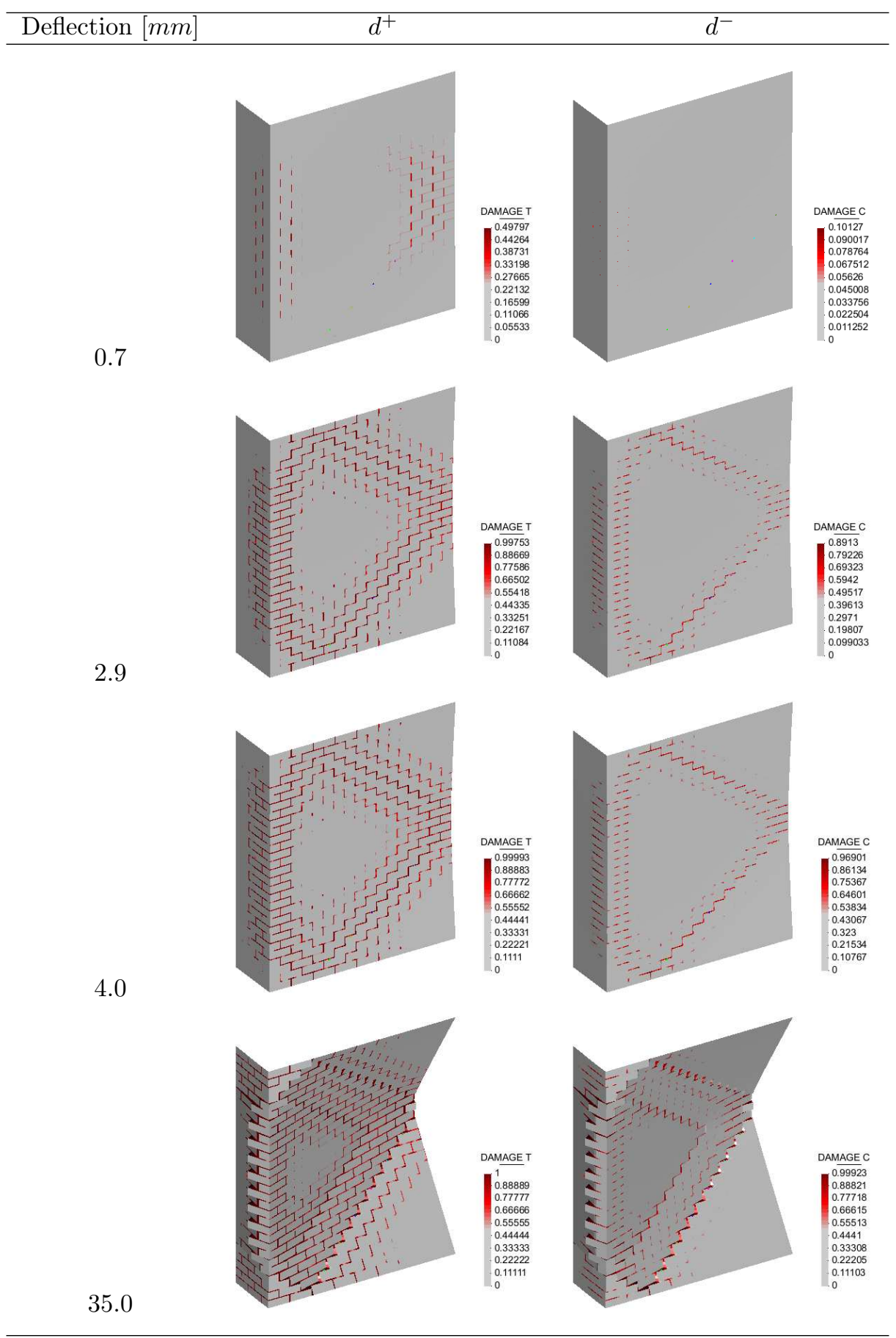

Table 2: Tensile and Compressive damage evolution at different load stages from micro-model analysis 
As a last note, the importance of adopting a 3D constitutive model instead of a planestress one, as already pointed out in Section 2.2, is clearly shown in Figure 19, where a detail of the bottom-left corner of the structure is given. The in-plane strain along local Xdirection at the top of the shell $\varepsilon_{x x(+H / 2)}=\varepsilon_{0, x x}+\kappa_{x x} \frac{H}{2} \cong 0.11+0.0026 \times 110 / 2=0.253$ is larger but comparable to the transverse shear strain $\varepsilon_{0, x z}=0.157$. It is evident that the transverse shear deformation cannot be neglected. The failure mode depicted in Figure 19 can be considered as an overall bending state of deformation along the local X-direction, if one thinks about the overall behavior of the "composite" masonry material. However, due to the arrangement of the micro-structure, and the strong difference in mechanical properties of units and mortar, a significant amount of transverse shear appears in the (softer) mortar joints, in order to allow units to bend around the vertical axis of the wall.

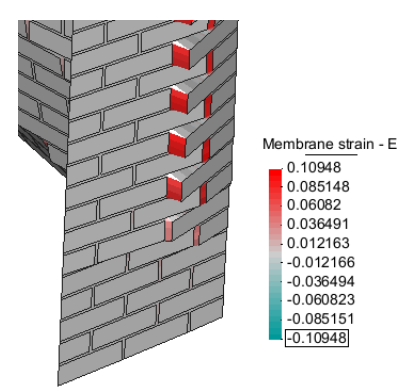

(a) $\varepsilon_{0, x x}$

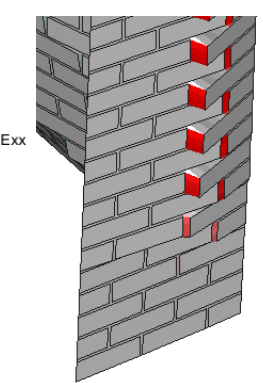

(b) $\kappa_{x x}$

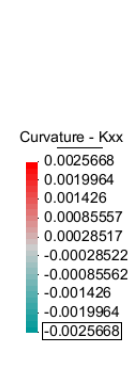
19964

Figure 19: Comparison of membrane, bending and shear strains in micro-model analysis

\subsection{Multiscale simulation}

In this section the proposed computational homogenization framework for shells is assessed, comparing it with the results obtained by the DNS presented in Section 4.4. For the multiscale simulations three macro-scale discretizations have been used, in order to check the regularization properties of the method with respect to the mesh size. It should be noted that the regularization procedure presented in [41] and briefly reported in Section 3 is only meant to provide objective results in terms of amount of dissipated energy with respect to the mesh size (i.e. to alleviate the mesh-size dependency). For problems related to mesh bias dependency $[62,63,64,12,65]$ (i.e. dependency on the mesh orientation), the adopted procedure should be accompanied with enhanced FE formulations at the macro-scale. In the present work, the used Shell FE has an enhancement in the membrane strain field following the EAS method [66], and in the transverse shear field following the MITC plate formulation $[67,68]$. The adopted element seems to be sufficiently accurate with respect to mesh orientation dependency. The adopted meshes are given in Figure 20, with a discretization size of $300 \mathrm{~mm}, 150 \mathrm{~mm}$ and $75 \mathrm{~mm}$ respectively. 


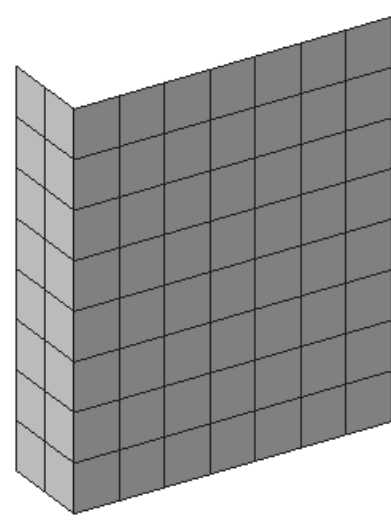

(a) $h=300 \mathrm{~mm}$

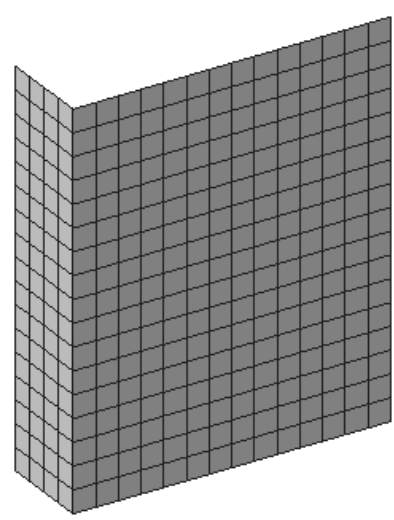

(b) $h=150 \mathrm{~mm}$

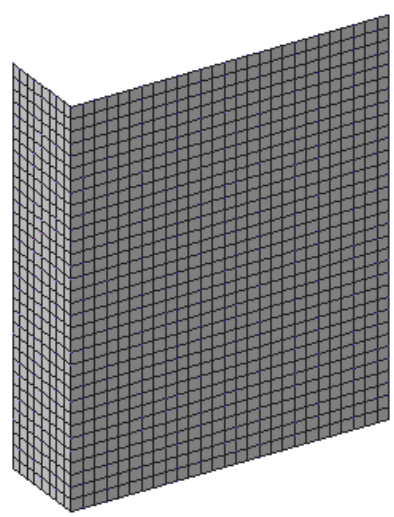

(c) $h=75 \mathrm{~mm}$

Figure 20: macro-scale meshes used for the multiscale simulations

Figure 21 shows the pressure-deflection curves obtained from the three multiscale analyses, compared to the DNS and experimental results.

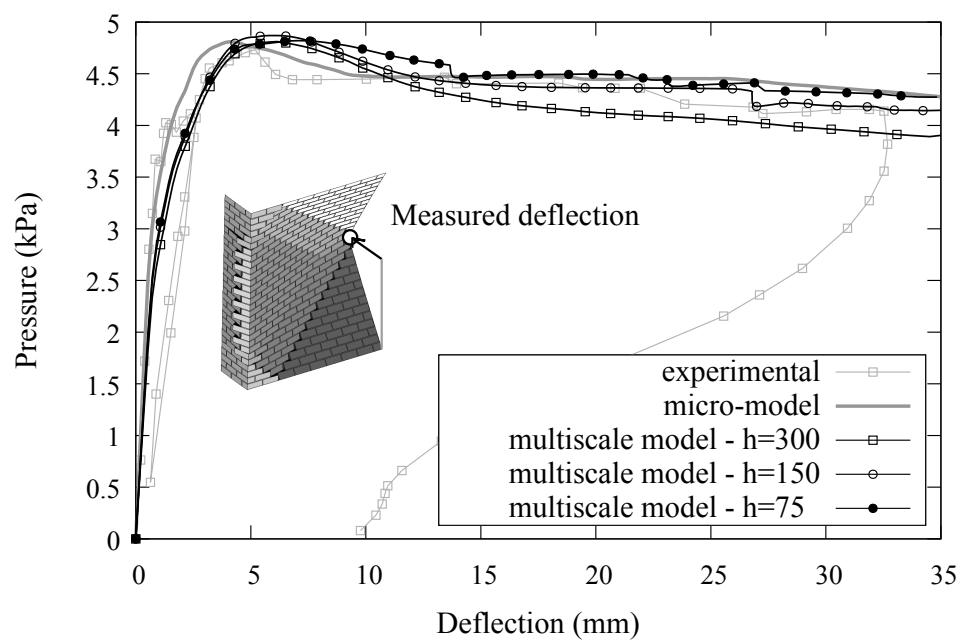

Figure 21: pressure-deflection curves obtained from the multiscale analyses

A satisfactory agreement can be seen between the homogenized responses and the micromodel response. Also the regularization of the response seems satisfactory, converging to a meaningful solution upon mesh refinement. A slight difference between the homogenized response and the DNS response can be seen for pressure values ranging from $3 \mathrm{kPa}$ 
to the peak load, with the homogenized models showing a softer response, thus leading to a slight overestimation of the displacement at peak strength, while the peak force is correctly predicted. This is believed to be due to the response given by connection (corner) between the main wall and the turning wall: in the micro-model, bricks from the front and lateral walls are physically connected. On the contrary, in the homogenized model this doesn't happen, with the RVEs being only weakly connected with one another (i.e. by means of the macroscopic homogenized strain), thus offering a slightly softer response. However, this initial small difference didn't seem to affect the overall response of the wall for the subsequent stages of the analysis.

Next, a detailed comparison between the DNS results and the Multiscale results is given. Figure 22 shows a comparison between the tensile and compressive damage patterns obtained from the three meshes used for the multiscale analyses, and those obtained from the DNS. The failure modes are in very good agreement with the predictions of the DNS, both in terms of crack-direction and in terms of initial spread of non-linearity (i.e. before localization into a well defined rotational hinge). Note that the damage index displayed in the homogenized results is defined as the volume average of the damage indexes in the RVE, thus can never reach the unity, unless all the elements in RVE are completely damaged. However this definition of damage has no physical meaning, and it has been used just for post-process purposes. Real damage values can be retrieved only from the RVE. The vertical hinge formed in the turning wall near the intersection with the front wall, developed with a narrow damaged zone, while the diagonal hinges on the front wall involved a significantly larger damaged zone. This behavior is mainly due to the redistribution of compressive stresses, initially uniform over the entire wall, and progressively increasing towards the center of the front wall, as shown in Figure 23. In fact, as shown in Figure 24, the moment curvature response monitored along the diagonal hinge (compared to the response monitored on the vertical hinge) showed a more ductile behavior with a considerable initial hardening, since the shell cross section was there subjected to significant compressive stresses. Figure 25 shows a comparison in terms of rotations and out-of-plane deflection. A remarkable agreement can be seen for rotations, both qualitatively and quantitatively, properly capturing the position and orientation of the vertical (along the corner) and diagonal (on the front face) rotational hinges, as predicted by the micro-model analysis. Figure 26 shows a comparison in terms of shell moments. In this case a satisfactory agreement is found in the overall bending moments distribution, while, obviously, from a quantitative point of view the peak values showed by the micro-model are smoothed-out in the multiscale model, since they are average values obtained from the homogenized response of the RVE.

Finally, Figure 27 shows a last comparison in terms of tensile damage obtained from the micro-model, the homogenized model, and the actual damage distribution in some relevant RVEs. It can be noticed how all the relevant failure modes highlighted by the micro-model are correctly captured by the homogenized one. RVE 1 shows a bending failure mechanism about the head joints direction. RVE 2, in the bottom-left corner of the wall, shows a transition from uniaxial bending about the head joints direction to diagonal bending. RVE 3 and RVE 4 show the damage pattern obtained from diagonal bending, with RVE 4 being on the localization band defined by the diagonal rotational hinge. RVE 5 , in the center of the wall, shows a bending failure mechanism about the bed joints direction. 

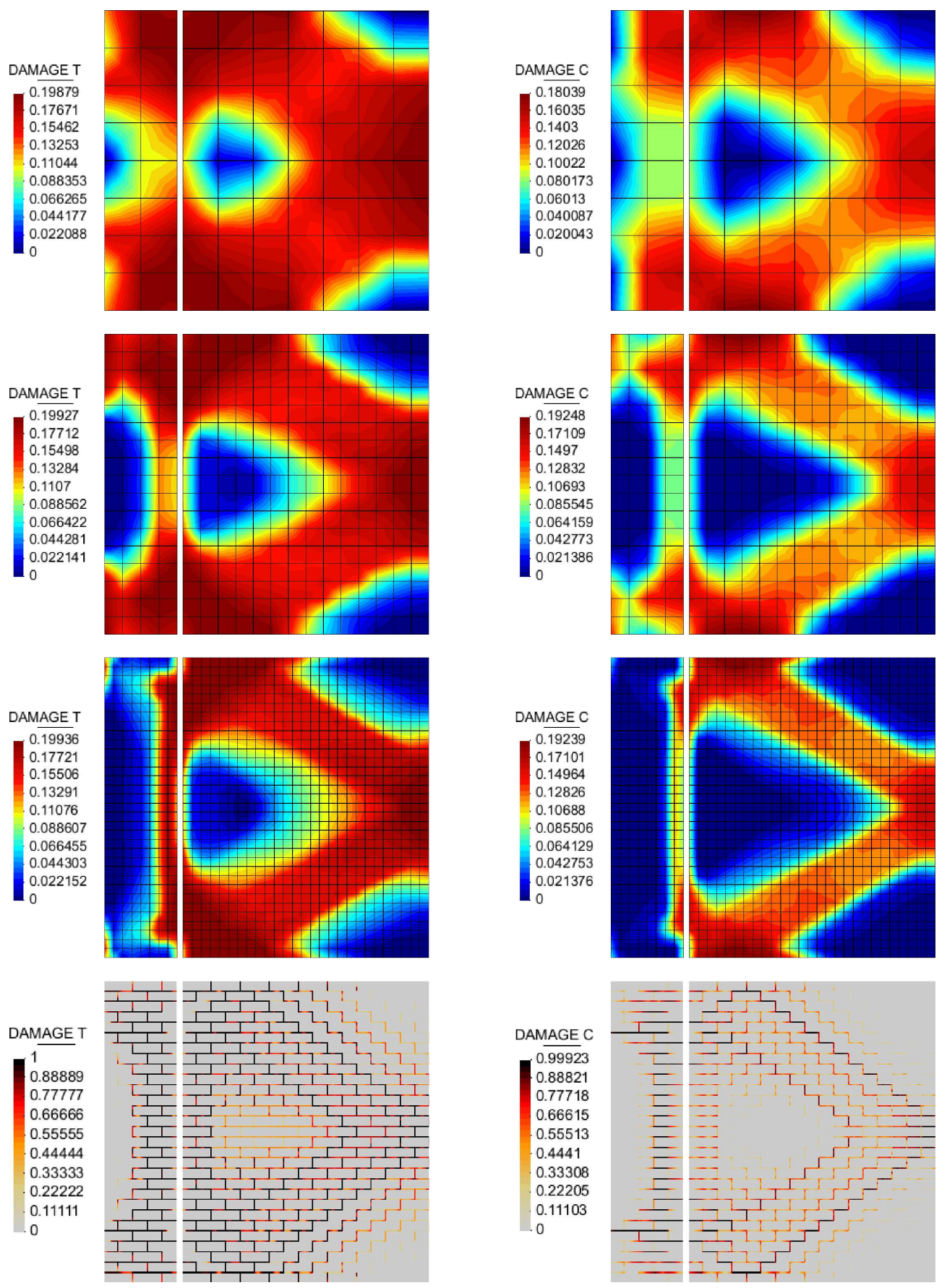

Figure 22: Comparison of tensile and compressive damage. Multiscale (first three rows) vs. DNS (last row) 

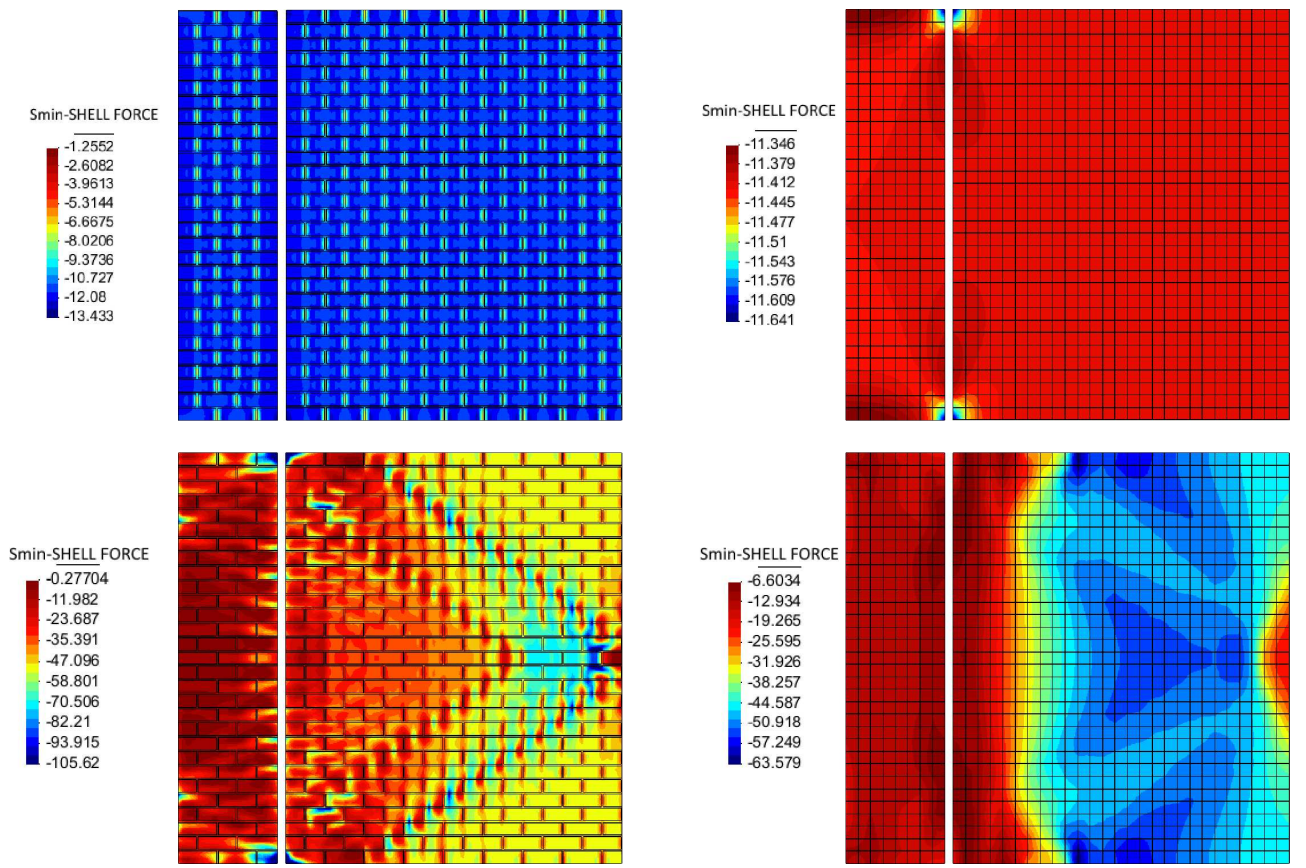

Figure 23: Comparison of minimum principal shell forces $[\mathrm{N} / \mathrm{mm}]$, after the initial pre-compression (row 1) and at the end of the analysis (row 2). DNS (column 1) vs. Multiscale (column 2).

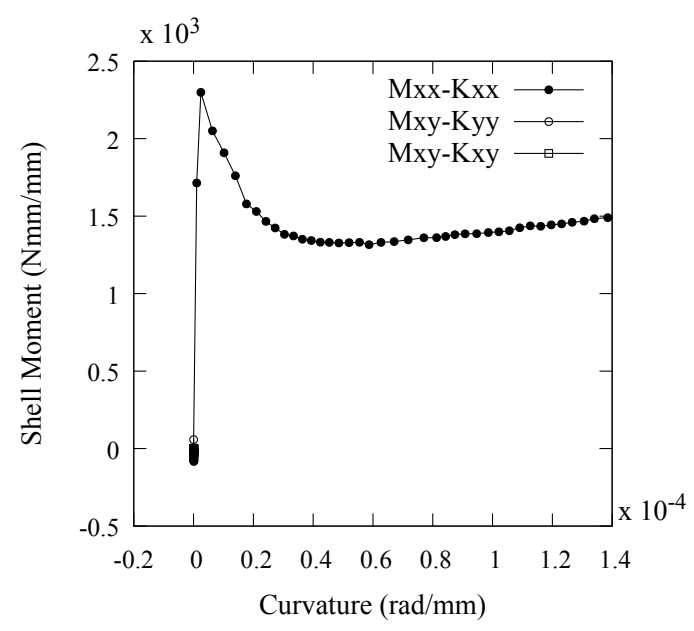

(a)

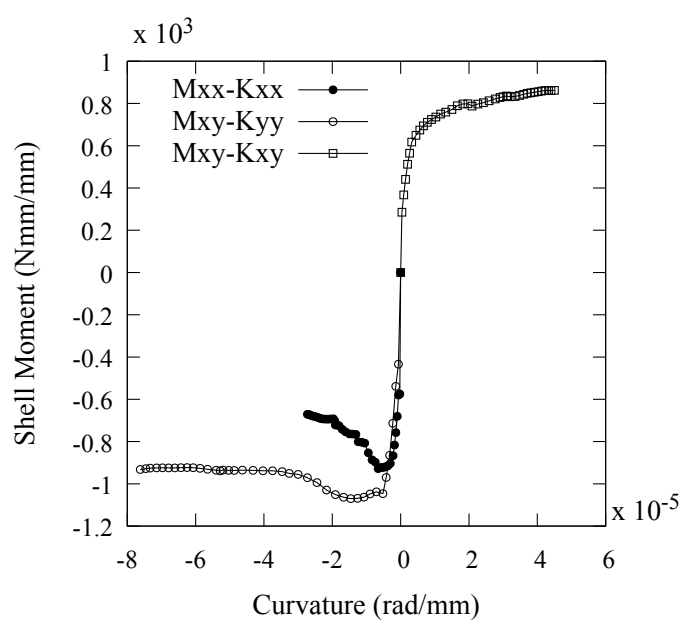

(b)

Figure 24: homogenized moment-curvature response: (a) at the center of the vertical hinge on the turning wall; (b) at the center of the bottom diagonal hinge on the front wall 

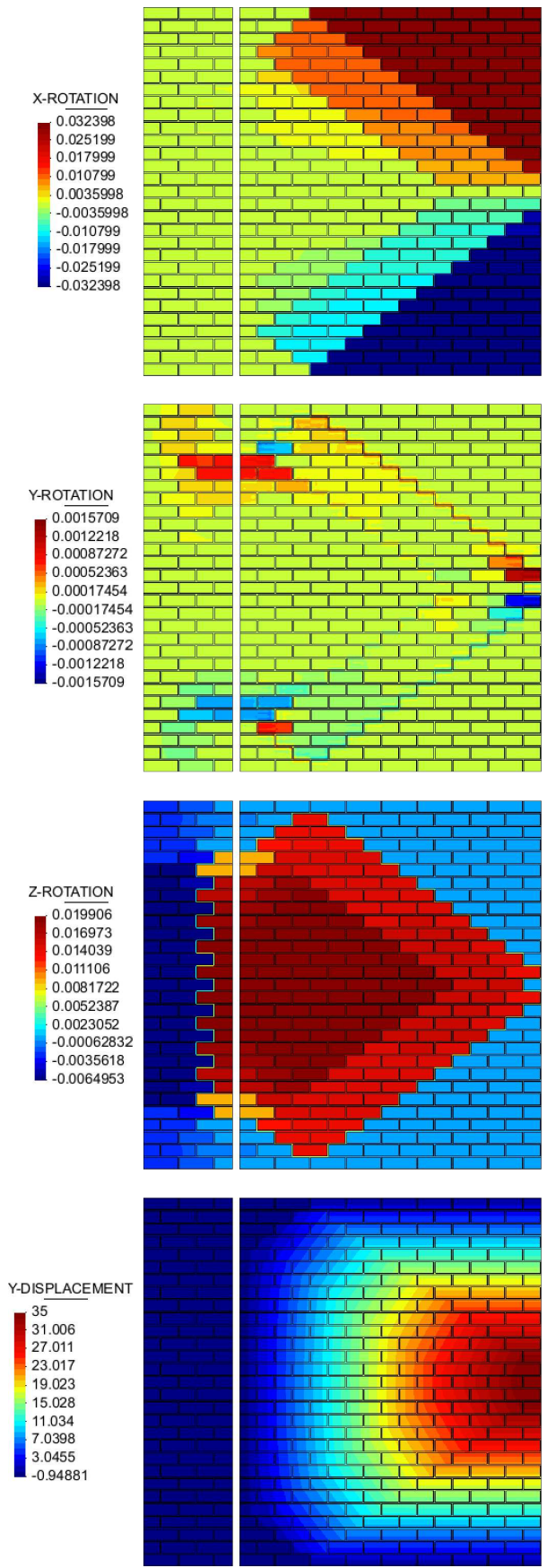

Figure 25: Comparison

Multiscale (column 2).
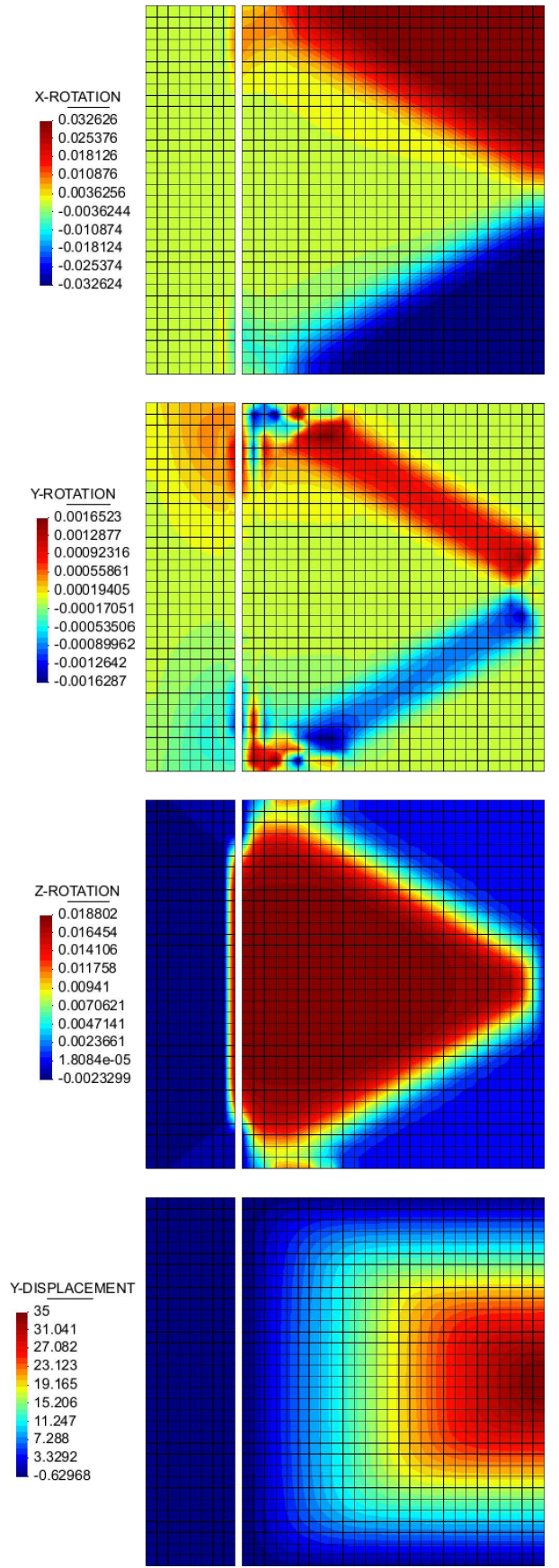

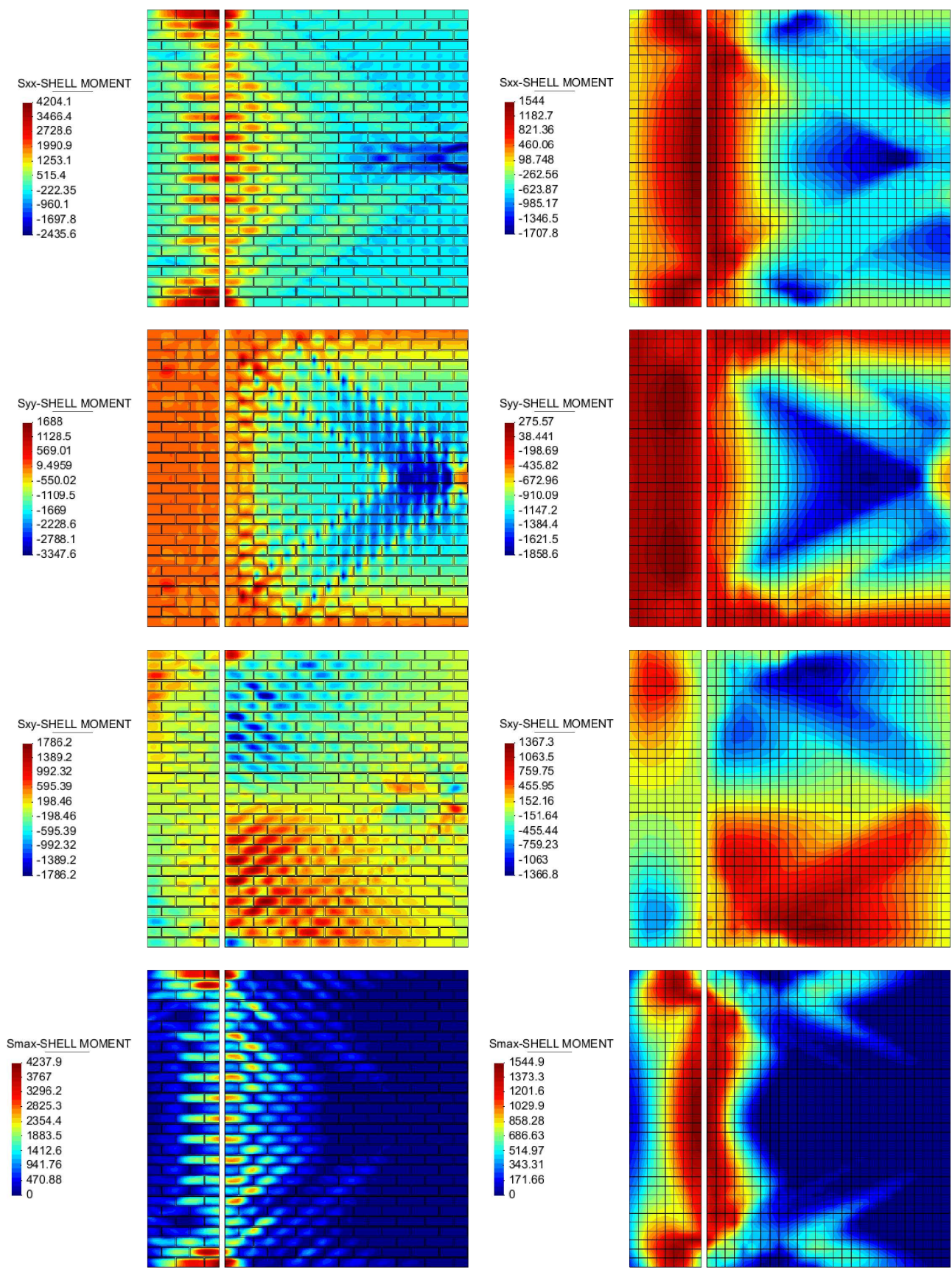

Figure 26: Comparison of shell moments [Nmm/mm]. DNS (column 1) vs. Multiscale (column 2). 


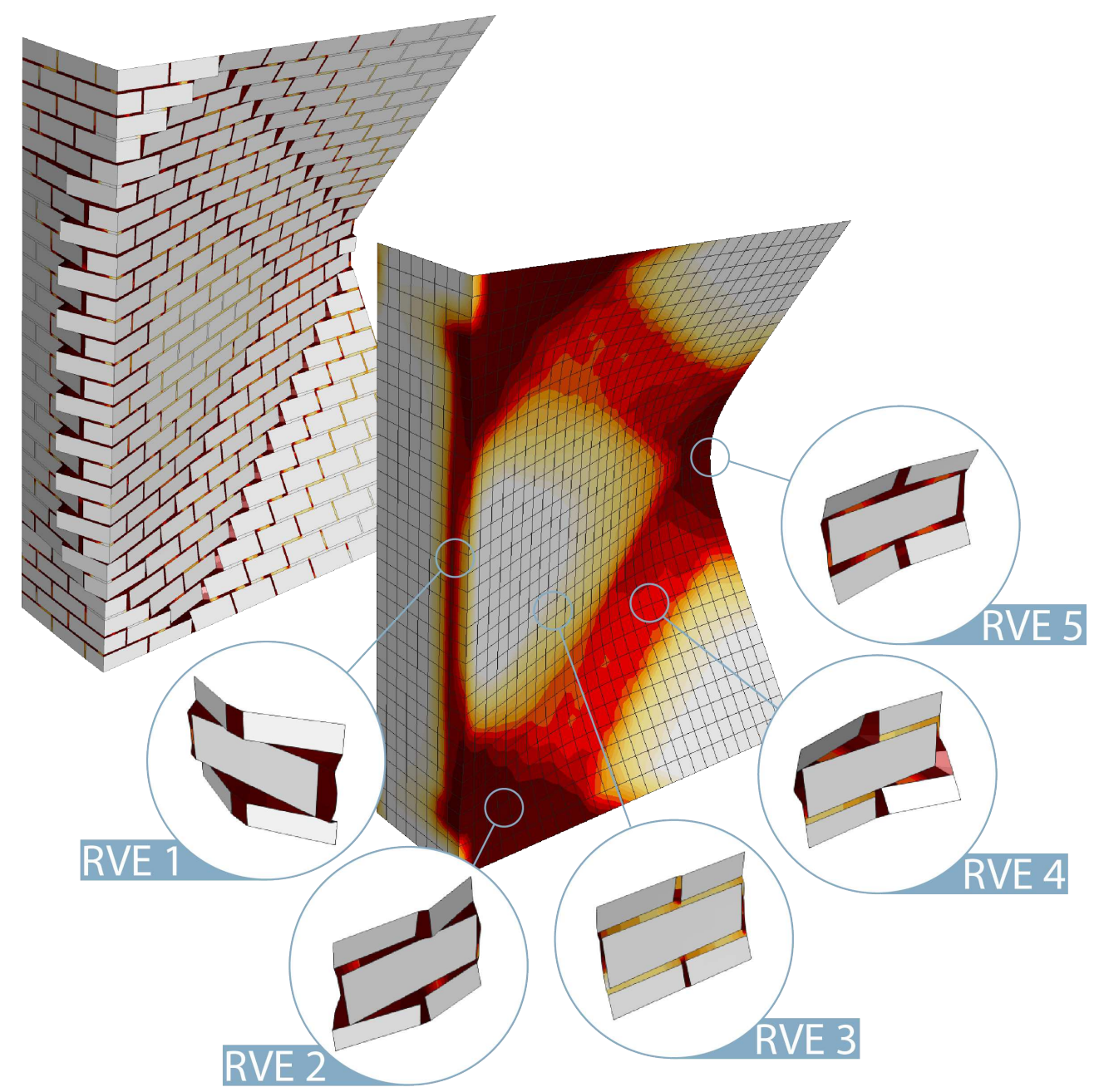

Figure 27: Distribution of tensile damage in some significant RVEs

\subsection{Computational cost of micro-scale and multi-scale simulations}

Even though computational performance is not the main topic of this work, a brief comparison between micro- and multi-scale analyses is given here in terms of computational costs. For both micro- and multi-scale analyses, the solution strategy consist of a first stage (from time $t=0$ to $t=0.02$ ), for the application of the vertical load, conducted under load control, and a second stage (from time $t=0.02$ to $t=1.0$ ), for the horizontal pressure, conducted under displacement control. An average time step $\Delta t=0.005$ is used. Equilibrium is attained within an average of three iterations per time step. In both stages, a full Newton-Raphson iterative procedure is used, thus updating the tangent matrix at each iteration. The analyses were carried out on a single machine with an 
Intel Core i7-2670QM-2.20GHz CPU and 8.00 GB RAM. The code is parallelized with OpenMP.

For the micro-model analysis, the average elapsed time per iteration is 00:00:43 [hh:mm:ss], and the total elapsed time is 07:12:27 [hh:mm:ss]. For the multiscale analysis with the coarsest discretization $(h=300 \mathrm{~mm})$, the average elapsed time per iteration is 00:00:29 [hh:mm:ss], and the total elapsed time is 04:54:30 [hh:mm:ss].

Overall, a slight speed-up from micro- to multi-scale modeling is obtained. Such a limited speed-up is probably due to the size of the masonry micro-structure, that is comparable with the FE size at the macro-scale. One could expect more benefits with smaller microstructures or with larger structural models. However, the computational cost for a full non-linear multiscale analysis is still too expensive for using this method with large scale simulations. It should be noted that in the present implementation, every integration point of the macro-scale has its own RVE (due to the non-linearity of the problem), and the multiscale simulation is carried out from the very beginning at every integration point. A substantial speed-up could be achieved using an adaptive triggering of RVE simulations only where and when needed, on the line of what has been recently proposed in [69].

\section{Comparison between DNS-3D DNS-shell and multiscale models.}

Section 4 presented an experimental-numerical comparison, used to assess the ability of the proposed framework to represent the behavior of masonry walls when loaded in the out-of-plane direction. This section gives a concluding example, where a 3D-based DNS, a shell-based DNS and a shell-based multiscale model are compared. This example is useful to assess the assumptions made in the shell model and in the shell-homogenization framework, and the capability of the proposed method to simulate the response of a full 3D model.

The example consists in a simply supported slab, vertically loaded in the center. Due to symmetry, only one half of the model is analyzed. The geometry is shown in Figure 28. The same model is analyzed with two DNS, 3D-based and shell-based, respectively (to assess the ability of the shell to simulate the 3D behavior), and with two shell-based multiscale simulations, using two discretization sizes of $250 \mathrm{~mm}$ and $125 \mathrm{~mm}$ respectively (to assess the regularization with respect to the macro-scale FE size).

Figure 29 shows the comparison of the load-deflection curves of the four analyses, while Figure 30 shows the damage pattern for the four analyses. A satisfactory agreement can be found between shell-based DNS and multiscale simulations, and the full 3D simulation. 


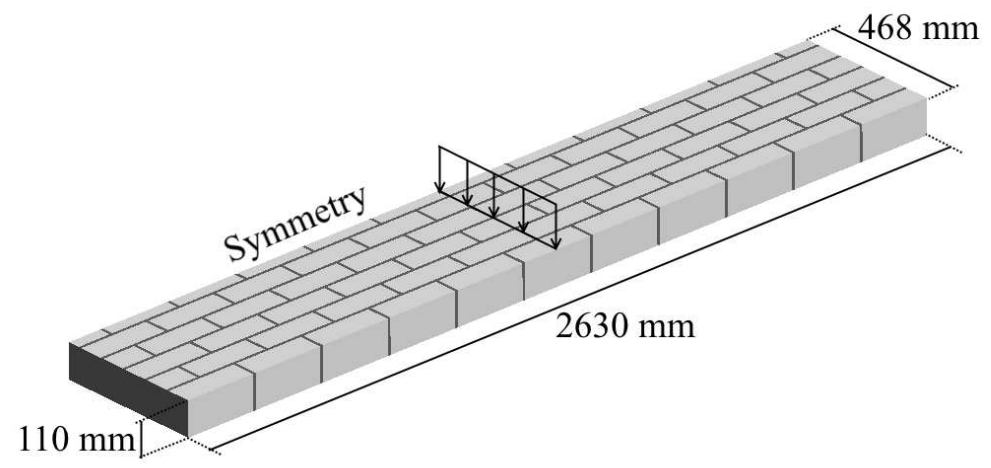

Figure 28: Geometry of the masonry slab. Only one half modeled due to symmetry

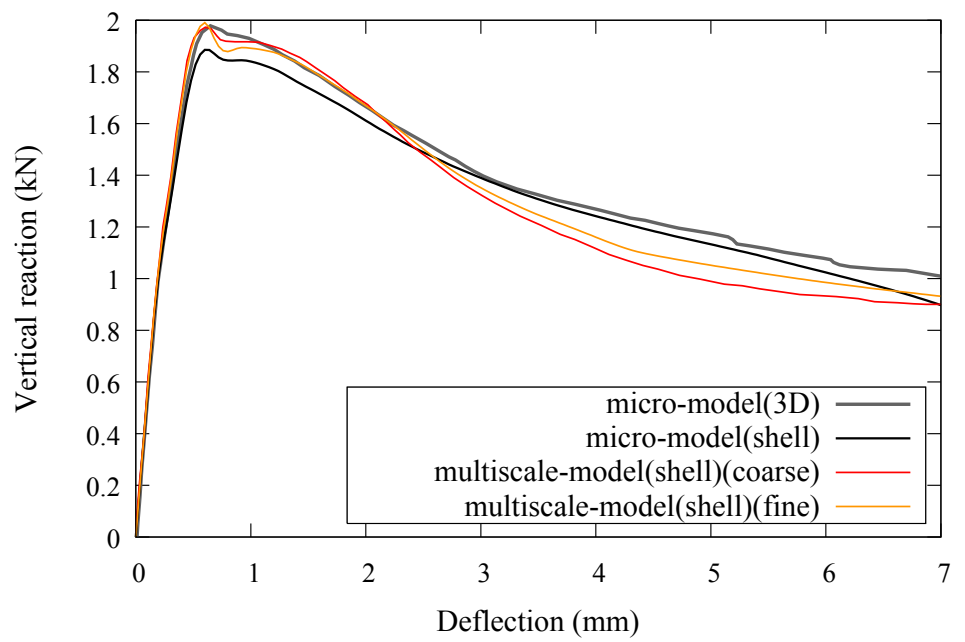

Figure 29: Load-deflection curves for the DNS and the multiscale models 


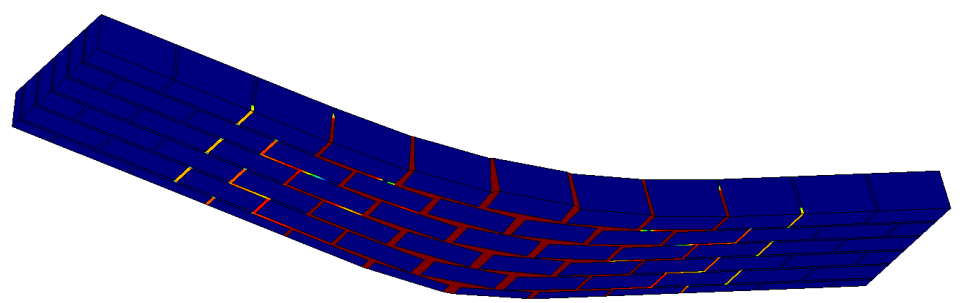

(a)

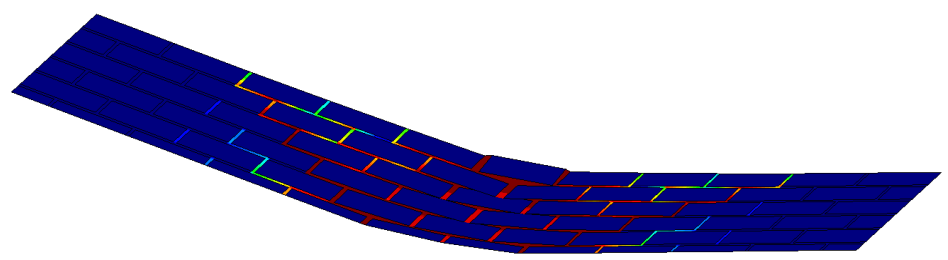

(b)

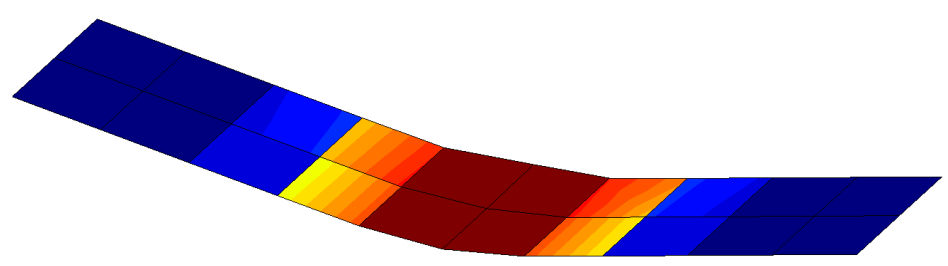

(c)

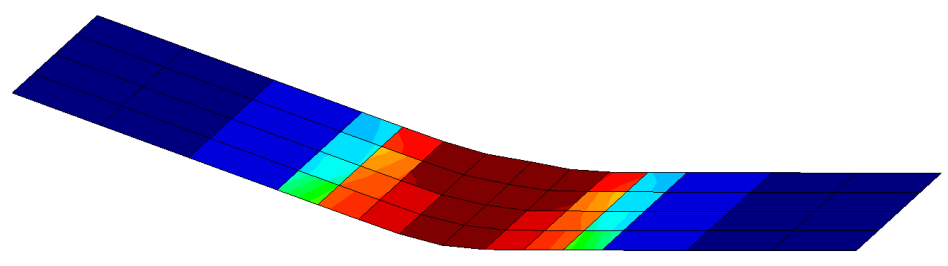

(d)

Figure 30: Tensile damage pattern for (a) 3D DNS, (b) Shell DNS, (c) Shell multiscale (coarse $h=$ $250 \mathrm{~mm}$ ), and (d) Shell multiscale (fine $h=125 \mathrm{~mm}$ ).

\section{Conclusions}

Classical computational homogenization is now an established tool for the analysis of complex heterogeneous structures. However, when dealing with thin shell-like structures, the characteristic size of the micro-structure may approach the thickness of the structural member, as it happens in one-leaf brick masonry walls, subject of the present study. In those cases, classical homogenization towards a three dimensional continuum is not applicable. 
This paper has proposed a computational homogenization method based on thick shell theory, able to provide an equivalent homogenous response in terms of generalized strains and generalized stresses, directly from the nested solution of a RVE. The method is designed to deal with micro-structures that can be considered as an extrusion of a heterogeneous surface (as in the case of one-leaf masonry walls), thus being heterogeneous in the in-plane directions, but (initially) homogeneous in the thickness direction. Under this hypothesis, both the macro-scale and the micro-scale RVE can be described by shell theories, greatly simplifying the problem of coupling micro and macro scales.

The proposed method has been applied to the analysis of unreinforced masonry walls undergoing out-of-plane loading. Experimental studies have been numerically reproduced to assess the capabilities of the method. The proposed methodology has proven to be rather accurate in reproducing the key aspects of the out-of-plane experimental behavior of URM walls, properly describing all the failure mechanisms beyond the point of maximum bending strength of the wall.

\section{Acknowledgments}

This research has received the financial support from the Graduate School of the University "G. d'Annunzio" of Chieti-Pescara, from the MINECO (Ministerio de Economia y Competitividad of the Spanish Government) through the MULTIMAS project (Multiscale techniques for the experimental and numerical analysis of the reliability of masonry structures, ref. num. BIA2015-63882-P) and the EACY project (Enhanced accuracy computational and experimental framework for strain localization and failure mechanisms, ref. MAT2013-48624-C2-1-P). Partial financial support by the ReLUIS (Network of Earthquake Engineering Experimental Laboratories, funded by the Prime Minister Office - Civil Protection Department) project is acknowledged and greatly appreciated. This publication, however, might not necessarily reflect the viewpoint of the Civil Protection Department.

S. Oller acknowledges the support of the European Research Council under the Advanced Grant: ERC-2012-AdG 320815 COMP-DES-MAT "Advanced tools for computational design of engineering materials" 


\section{References}

[1] L. Pelà, M. Cervera, P. Roca, Continuum damage model for orthotropic materials: Application to masonry, Computer Methods in Applied Mechanics and Engineering 200 (9-12) (2011) 917-930, ISSN 0045-7825, URL http://www.sciencedirect.com/science/article/pii/S0045782510003257.

[2] L. Pelà, M. Cervera, P. Roca, An orthotropic damage model for the analysis of masonry structures, Construction and Building Materials 41 (2013) 957-967, ISSN 0950-0618, URL http: //www.sciencedirect.com/science/article/pii/S0950061812004837.

[3] P. Roca, M. Cervera, G. Gariup, L. Pelà, Structural Analysis of Masonry Historical Constructions. Classical and Advanced Approaches, Archives of Computational Methods in Engineering 17 (3) (2010) 299-325, ISSN 1134-3060.

[4] P. B. Lourenço, J. G. Rots, Multisurface interface model for analysis of masonry structures, Journal of engineering mechanics 123 (7) (1997) 660-668.

[5] P. B. Lourenco, Computational strategies for masonry structures, Ph.D. thesis, TU Delft, Delft University of Technology, 1996.

[6] D. V. Oliveira, P. B. Lourenço, Implementation and validation of a constitutive model for the cyclic behaviour of interface elements, Computers \& structures 82 (17) (2004) 1451-1461.

[7] A. Drougkas, L. Pelà, P. Roca, Numerical modelling of masonry shear walls failure mechanisms, in: Proceedings of 9th International Masonry Conference, Guimarães, Portugal, 2014.

[8] C. Citto, Two-dimensional interface model applied to masonry structures, ProQuest, 2008.

[9] L. Gambarotta, S. Lagomarsino, Damage models for the seismic response of brick masonry shear walls. Part I: The mortar joint model and its applications, Earthquake Engineering \& Structural Dynamics 26 (4) (1997) 423-439, ISSN 1096-9845.

[10] M. L. Raffa, F. Lebon, E. Sacco, H. Welemane, A multi-level interface model for damaged masonry, in: E. B.H.V. Topping, P. Iv/'anyi (Ed.), "Proceedings of the Fourteenth International Conference on Civil, Structural and Environmental Engineering Computing", Civil-Comp Press, Stirlingshire, UK, 2013.

[11] E. Sacco, J. Toti, Interface Elements for the Analysis of Masonry Structures, International Journal for Computational Methods in Engineering Science and Mechanics 11 (6) (2010) 354-373, URL http://www.tandfonline.com/doi/abs/10.1080/15502287.2010.516793.

[12] L. Pelà, M. Cervera, S. Oller, M. Chiumenti, A localized mapped damage model for orthotropic materials, Engineering Fracture Mechanics 124-125 (2014) 196-216, ISSN 0013-7944, URL http: //www.sciencedirect.com/science/article/pii/S0013794414001386.

[13] P. B. Lourenço, Anisotropic softening model for masonry plates and shells, Journal of Structural Engineering 126 (9) (2000) 1008-1016.

[14] D. Addessi, E. Sacco, A. Paolone, Cosserat model for periodic masonry deduced by nonlinear homogenization, European Journal of Mechanics-A/Solids 29 (4) (2010) 724-737.

[15] E. Bosco, V. Kouznetsova, M. Geers, Multi-scale computational homogenization-localization for propagating discontinuities using X-FEM, International Journal for Numerical Methods in Engineering 102 (3-4) (2015) 496-527.

[16] M. L. De Bellis, A Cosserat based multi-scale technique for masonry structures, in: PHD Thesis, 2009.

[17] M. L. De Bellis, D. Addessi, A Cosserat based multi-scale model for masonry structures, International Journal for Multiscale Computational Engineering 9 (5).

[18] E. A. D. S. Neto, R. A. Feijóo, Variational Foundations of Multi-Scale Constitutive Models of Solid: Small and Large Strain Kinematical Formulation, LNCC Research and Development (16).

[19] M. Geers, V. G. Kouznetsova, W. A. M. Brekelmans, MultiScale First-Order and Second-Order Computational Homogenization of Microstructures towards Continua, International Journal for Multiscale Computational Engineering 1 (4), ISSN 1543-1649.

[20] J. A. Hernández, J. Oliver, A. E. Huespe, M. Caicedo, High-Performance Model Reduction Procedures in Multiscale Simulations, 127, Monograph CIMNE, 2012.

[21] V. Kouznetsova, M. Geers, W. Brekelmans, Multi-scale second-order computational homogenization of multi-phase materials: a nested finite element solution strategy, Computer Methods in Applied Mechanics and Engineering 193 (2004) 5525-5550, ISSN 0045-7825.

[22] V. Kouznetsova, M. G. D. Geers, W. A. M. Brekelmans, Multi-scale constitutive modelling of heterogeneous materials with a gradient-enhanced computational homogenization scheme, International Journal for Numerical Methods in Engineering 54 (8) (2002) 1235-1260, ISSN 1097-0207.

[23] T. J. Massart, Multi-scale modeling of damage in masonry structures .

[24] T. Massart, R. Peerlings, M. Geers, An enhanced multi-scale approach for masonry wall compu- 
tations with localization of damage, International Journal for Numerical Methods in Engineering 69 (5) (2007) 1022-1059.

[25] B. Mercatoris, P. Bouillard, T. Massart, Multi-scale detection of failure in planar masonry thin shells using computational homogenisation, Engineering fracture mechanics 76 (4) (2009) 479-499.

[26] B. Mercatoris, T. Massart, A coupled two-scale computational scheme for the failure of periodic quasi-brittle thin planar shells and its application to masonry, International journal for numerical methods in engineering 85 (9) (2011) 1177-1206.

[27] X. Oliver Olivella, M. A. Caicedo Silva, E. Roubin, A. E. Huespe, et al., Continuum approach to computational multi-scale modeling of fracture, Key engineering materials 627 (2014) 349-352.

[28] J. Oliver, I. Dias, A. E. Huespe, Crack-path field and strain-injection techniques in computational modeling of propagating material failure, Computer Methods in Applied Mechanics and Engineering 274 (2014) 289-348.

[29] J. Oliver, M. Caicedo, E. Roubin, J. Hernández, A. Huespe, Multi-scale (FE2) analysis of material failure in cement/aggregate-type composite structures, Computational Modelling of Concrete Structures (2014) 39.

[30] S. Oller, J. Miquel Canet, F. Zalamea, Composite material behavior using a homogenization double scale method, Journal of Engineering mechanics 131 (1) (2005) 65-79.

[31] J. M. Ortolano, J. A. Hernández, J. Oliver, A Comparative Study on Homogenization Strategies for Multi-Scale Analysis of Materials, 135, Monograph CIMNE, 2013.

[32] F. Otero, X. Martínez, S. Oller, O. Salomón, Study and prediction of the mechanical performance of a nanotube-reinforced composite, Composite Structures 94 (2012) 2920-2930.

[33] F. Otero, S. Oller, X. Martinez, O. Salomón, Numerical homogenization for composite materials analysis. Comparison with other micro mechanical formulations, Composite Structures 122 (2015) $405-416$.

[34] H. A. Badillo, Numerical modelling based on the multiscale homogenization theory. Application in composite materials and structures, Ph.D. thesis, Universitat Politécnica de Catalunya BarcelonaTech, 2012.

[35] R. D. Quinteros, S. Oller, L. G. Nallim, Nonlinear homogenization techniques to solve masonry structures problems, Composite Structures 94 (2) (2012) 724-730.

[36] P. Trovalusci, R. Masiani, Non-linear micropolar and classical continua for anisotropic discontinuous materials, International Journal of Solids and Structures 40 (5) (2003) 1281-1297.

[37] A. Zucchini, P. Lourenço, A micro-mechanical model for the homogenisation of masonry, International Journal of Solids and Structures 39 (12) (2002) 3233-3255.

[38] A. Zucchini, P. B. Lourenço, A micro-mechanical homogenisation model for masonry: Application to shear walls, International Journal of Solids and Structures 46 (3) (2009) 871-886.

[39] V. P. Nguyen, M. Stroeven, L. J. Sluys, Multiscale continuous and discontinuous modeling of heterogeneous materials: A review on recent developments, Journal of Multiscale Modelling 3 (04) (2011) 229-270.

[40] T. Belytschko, S. Loehnert, J.-H. Song, Multiscale aggregating discontinuities: a method for circumventing loss of material stability, International Journal for Numerical Methods in Engineering 73 (6) (2008) 869-894.

[41] M. Petracca, L. Pelà, R. Rossi, S. Oller, G. Camata, E. Spacone, Regularization of first order computational homogenization for multiscale analysis of masonry structures, Computational Mechanics 57 (2) (2016) 257-276, ISSN 1432-0924, URL http://dx.doi.org/10.1007/s00466-015-1230-6.

[42] M. Geers, E. Coenen, V. Kouznetsova, Multi-scale computational homogenization of structured thin sheets, Modelling and Simulation in Materials Science and Engineering 15 (4) (2007) S393.

[43] M. Geers, V. Kouznetsova, W. Brekelmans, Multi-scale computational homogenization: Trends and challenges, Journal of computational and applied mathematics 234 (7) (2010) 2175-2182.

[44] E. Coenen, V. Kouznetsova, M. Geers, Computational homogenization for heterogeneous thin sheets, International Journal for Numerical Methods in Engineering 83 (8-9) (2010) 1180-1205.

[45] Y. Cong, S. Nezamabadi, H. Zahrouni, J. Yvonnet, Multiscale computational homogenization of heterogeneous shells at small strains with extensions to finite displacements and buckling, International Journal for Numerical Methods in Engineering 104 (4) (2015) 235-259.

[46] R. Grytz, G. Meschke, Computational homogenization in multi-scale shell analysis at large strains, in: III European Conference on Computational Mechanics (ECCM 2006), 592, 2006.

[47] M. Griffith, J. Vaculik, Out-of-plane flexural strength of unreinforced clay brick masonry walls, TMS Journal 25 (1) (2007) 53-68.

[48] G. Cowper, The shear coefficient in Timoshenko's beam theory, Journal of applied mechanics 33 (2) (1966) 335-340. 
[49] R. De Borst, The zero-normal-stress condition in plane-stress and shell elastoplasticity, Communications in applied numerical methods 7 (1) (1991) 29-33.

[50] R. Hill, A self-consistent mechanics of composite materials, Journal of the Mechanics and Physics of Solids 13 (4) (1965) 213-222.

[51] J. Mandel, Plasticité classique et viscoplasticité, Springer-Verlag, 1971.

[52] P. Dadvand, R. Rossi, E. Oñate, An object-oriented environment for developing finite element codes for multi-disciplinary applications, Archives of computational methods in engineering 17 (3) (2010) 253-297.

[53] P. Dadvand, R. Rossi, M. Gil, X. Martorell, J. Cotela, E. Juanpere, S. R. Idelsohn, E. Oñate, Migration of a generic multi-physics framework to HPC environments, Computers \& Fluids 80 (2013) 301-309.

[54] GiD: The personal pre and post preprocessor, 2002.

[55] M. Cervera, J. Oliver, R. Faria, Seismic evaluation of concrete dams via continuum damage models, Earthquake engineering \& structural dynamics 24 (9) (1995) 1225-1245.

[56] R. Faria, J. Oliver, M. Cervera, A strain-based plastic viscous-damage model for massive concrete structures, International Journal of Solids and Structures 35 (14) (1998) 1533-1558.

[57] J. Y. Wu, J. Li, R. Faria, An energy release rate-based plastic-damage model for concrete, International Journal of Solids and Structures 43 (3) (2006) 583-612.

[58] J. Lubliner, J. Oliver, S. Oller, E. Oñate, A plastic-damage model for concrete, International Journal of solids and structures 25 (3) (1989) 299-326.

[59] Z. Bažant, B. Oh, Crack Band Theory for Fracture of Concrete, Bordas-Dunod, URL http:// books.google.it/books?id=x9citwAACAAJ, 1983.

[60] J. Oliver, A consistent characteristic length for smeared cracking models, International Journal for Numerical Methods in Engineering 28 (2) (1989) 461-474.

[61] S. Oller, Fractura Mecánica: Un Enfoque Global, Ediciones CIMNE y UPC, 2001.

[62] M. Cervera, M. Chiumenti, R. Codina, Mixed stabilized finite element methods in nonlinear solid mechanics. Part II: Strain Localization, Computer Methods in Applied Mechanics and Engineering 199 (37) (2010) 2571-2589.

[63] L. Benedetti, M. Cervera, M. Chiumenti, Stress-accurate Mixed FEM for soil failure under shallow foundations involving strain localization in plasticity, Computers and Geotechnics 64 (2015) 32-47.

[64] M. Cervera, L. Pelà, R. Clemente, P. Roca, A crack-tracking technique for localized damage in quasi-brittle materials, Engineering Fracture Mechanics 77 (13) (2010) 2431-2450.

[65] S. Saloustros, L. Pelà, M. Cervera, A crack-tracking technique for localized cohesive-frictional damage, Engineering Fracture Mechanics 150 (2015) 96-114, ISSN 0013-7944, URL http://dx.doi. org/10.1016/j.engfracmech.2015.10.039.

[66] J. C. Simo, M. Rifai, A class of mixed assumed strain methods and the method of incompatible modes, International Journal for Numerical Methods in Engineering 29 (8) (1990) 1595-1638.

[67] E. N. Dvorkin, K.-J. Bathe, A continuum mechanics based four-node shell element for general non-linear analysis, Engineering computations 1 (1) (1984) 77-88.

[68] K.-J. Bathe, E. N. Dvorkin, A four-node plate bending element based on Mindlin/Reissner plate theory and a mixed interpolation, International Journal for Numerical Methods in Engineering 21 (2) (2005) 367-383.

[69] F. Otero, X. Martinez, S. Oller, O. Salomón, An efficient multi-scale method for non-linear analysis of composite structures, Composite Structures 131 (2015) 707-719. 